\title{
Der allgegenwärtige Wettstreit in den Künsten der Frühen Neuzeit
}

Jan-Dirk Müller/Ulrich Pfisterer

\section{Wettstreit als frühneuzeitliche Epochensignatur}

Aemulatio ist ein Zentralbegriff frühneuzeitlicher Ästhetik; ${ }^{1}$ er schließt einen Überbietungsgestus ein. Im Rahmen der Nachahmungspoetik ${ }^{2}$ tritt jeder neue Text, jedes neue Kunstwerk nicht nur in die Nachfolge älterer Vorbilder, sondern auch in Wettstreit mit ihnen. Ziel ist es, das Vorbild nicht nur zu erreichen, sondern möglichst zu übertreffen. Der frühneuzeitlichen Ästhetik eignet deshalb ein agonales Prinzip. Dieses agonale Prinzip reicht über den Bereich der Künste im engeren Sinne hinaus; es kennzeichnet schon die höfische Kultur des Mittelalters, nimmt aber in der aristokratisch geprägten Kultur der Frühen Neuzeit eine besondere Gestalt an (vgl. die einleitenden Überlegungen von Eric Achermann). ${ }^{3}$

Es war diese Beobachtung, die am Anfang des Plans stand, im Rahmen des Münchner SFB 573 'Pluralisierung und Autorität in der Frühen Neuzeit' ${ }^{\prime 4}$ eine Tagung über aemulatio in der Frühen Neuzeit zu veranstalten. Die gemeinsame rhetorische Basis der frühneuzeitlichen Kultur legte es zwar nahe, die Überlegungen in Literatur und bildender Kunst zum Prinzip der aemulatio zum Ausgangspunkt zu machen. Diese blieben der disziplinäre Kern des Tagungsprogramms, aber es war von vornherein die Absicht, den Untersuchungsrahmen nicht auf (literarische und bildkünstlerische) Rhetorik und Poetik einzuschränken, sondern zu fragen, ob man aemulatio nicht im Sinne Michel Foucaults als Dispositiv ${ }^{5}$ auffassen kann, das die frühneuzeitliche Kultur auch in anderen Lebensbereichen

1 Bauer 1992.

2 Kaminski 1998.

3 Diese Einsicht ist geeignet, die unfruchtbare Diskussion über den noch-mittelalterlichen oder schon-neuzeitlichen Charakter des 16. Jahrhunderts auf eine neue Grundlage zu stellen; vgl. Müller, J.-D. 1982.

4 Vgl. zu den Zielen des letzten Untersuchungszeitraums Antragsbuch 2007.

5 Foucault 1973. 
prägt. Aus diesem Grunde leitet den Band eine Sektion ein, die fragt, ob aemulatio nicht als Epochensignatur zu verstehen ist, welche die agonale höfische Kultur, deren Spiegel in humanistischer Panegyrik und den Wettstreit um künstlerische und literarische Vollkommenheit miteinander verbindet (vgl. besonders Maurice $\mathrm{Sa}$ in diesem Band).

In diese Richtung deuten bereits die Überlegungen Jacob Burckhardts Cultur der Renaissance in Italien (1860), wo - zwei Jahre nachdem Darwins Evolutionstheorie den dauernden Kampf der „Geeignetsten“ ums Überleben zum naturgegebenen Fundamentalprinzip erhoben hatte - für das Italien der Jahrzehnte um 1500 „der Krieg als Kunstwerk“ charakterisiert wird (Kap. I.9). ${ }^{6} \mathrm{Da}$ die wenigen Bruchstücke Burckhardts für den geplanten, eigentlichen Hauptband zur 'Kunst der Renaissance in Italien' nur annähernde Einblicke in Inhalt und Aufbau erlauben, lässt sich umso leichter spekulieren und behaupten, dass dort zumindest ein Kapitel mit 'Die Kunst als Krieg' überschrieben gewesen wäre. Bei allem Erkenntnisgewinn seitdem zu Einzelfragen aus dem weiten Feld der aemulatio in den Künsten steht eine umfassende Untersuchung des Phänomens immer noch aus.

Eine solche Fragestellung ist natürlich vor einer Beliebigkeit der Gegenstände zu bewahren. Schließlich ist Agonalität eine Voraussetzung jeder Adelskultur, und wenn man die Frage auf Rivalität und Konkurrenz ausweitet, dann dürfte es kaum eine Zeit geben, die nicht unter diesem Gesichtspunkt zu betrachten wäre. Anders ist das, wenn man mit dem rhetorischen Begriff der aemulatio die ästhetischen Komponenten des Wettstreits fokussiert, denn im Unterschied zur Moderne haben sich zumindest im frühneuzeitlichen Denk- und Theorierahmen - Kunst und Literatur noch nicht aus dem umfassenderen Zusammenhang einer Ästhetik des Lebens verabschiedet. Nicht auf das Mehr, das Effizienter, das Brauchbarer, das Lukrativer, das Dauerhafter usw. kommt es demnach an, sondern auf das Schöner und Vollkommener. ${ }^{7}$ So präzisiert erweist sich das Prinzip der aemulatio als ein Schlüssel für die Erkenntnis scheinbar sehr weit auseinander liegender Phänomene, und es strahlt auch in Bereiche wie

6 Vgl. für die Fragmente Burckhardt 2006, zu einigen hier interessierenden Aspekten Erben 2002 sowie Reinhardt 2004.

7 Bezeichnend der 1441 von Leon Battista Alberti in Florenz organisierte Certame Coronario, mit dem die Idealform des Wettstreits wiederbelebt werden sollte, ein Dichter-Agon all'antica, bei dem es (theoretisch) allein um den freundschaftlichen, gegenseitigen Ansporn zu tugendhafter Vervollkommnung (letztlich im Sinne Platons) ging; dazu Gorni 1972; Baader 2007. Vgl. zum Grundprinzip auch Delaney 1993-1994. 
die frühneuzeitliche Wissenschaft aus, die in der Moderne ganz anderen Funktionsprimaten unterworfen ist.

Eine systematische Vermessung des Feldes von aemulatio ist bei dieser Sachlage noch nicht möglich, und definitorische Bestimmungen wären eher kontraproduktiv, gilt es doch zunächst einmal, die üblichen disziplinären Grenzen zu öffnen und die Tauglichkeit des rhetorischen und kunsttheoretischen Konzepts jenseits dieser Grenzen zu erproben. Am Ausgangspunkt des Kolloquiums stand also der Gedanke, dass die spezifisch ästhetischen Phänomene frühneuzeitlicher aemulatio nicht allein vor einem literatur- und kunstimmanenten Horizont in den Griff zu bekommen sind, wenn sie auch dort besonders plakativ hervortreten. So finden sich in den bildenden Künsten besonders eindrückliche Beispiele für die Verbindung des ästhetischen Prinzips der aemulatio mit Wettstreit, Konkurrenz, Rivalität. Beim Großauftrag für die zweiten Bronzetüren des Florentiner Baptisteriums 1401 wurde etwa das Prinzip einer offenbar italienweiten Ausschreibung und eines anschließenden Wettbewerbs anhand von Probestücken eingeführt. ${ }^{8}$ Wenn dann gut einhundert Jahre später die Florentiner Signoria in der Sala dei Cinquecento des Palazzo Vecchio Michelangelo und Leonardo da Vinci beauftragt, an gegenüberliegenden Wänden je eine Kampfszene der jüngeren Vergangenheit zu malen, wird die Konkurrenz nicht mehr nur ins Vorfeld des (fertigen) Werkes verlegt, sondern als dessen bleibender Bestandteil richtiggehend inszeniert. Diese Formen der Qualitätssteigerung durch mehr oder weniger institutionalisierten Wettbewerb, aber auch das öffentliche Austragen von Künstler-(Wett-)Streiten kamen jedenfalls schnell auch an anderen Orten zum Einsatz.

Die Veränderungen in der Künstlersoziologie arbeiteten dieser Agonalisierung noch zu: Es eröffneten sich neue Freiräume außerhalb der etablierten Zünfte und Gilden. Herrscher nutzten die Ernennung von Hofkünstlern immer mehr dazu, herausragende Künstler zumindest teilweise an ihren Hof zu binden und über deren unverkennbares künstlerisches Profil das eigene Ansehen zu steigern. Ähnliche Intentionen konnten gleichzeitig bürgerliche Mäzene verfolgen. Diese neue Freiheit und die Ausbildung eines freien Marktes erforderten im Gegenzug von den Künstlern aber auch in neuem Maße, sich selbst zu präsentieren, zu 'ver-

8. Middeldorf-Kosegarten 1980. Zum größeren Kontext Prochno 2006 und Kanz 2011.

9 Etwa Wittkower 1989, 245-268; Goffen 2002; vgl. für Venedig Ilchman 2009 sowie Acidini 2009. 
markten' und auch auf die neuen Zwänge zu reagieren. ${ }^{10}$ Der zunehmende Wille und zugleich die Notwendigkeit zu individueller Einmaligkeit, Wiedererkennbarkeit und Abgrenzung führten unweigerlich zu wettstreitendem Konkurrieren um begrenzten Ruhm, begrenzte Posten, Aufträge und finanzielle Mittel.

Gefördert wird der Wettstreit durch die seit dem 15. Jahrhundert schnell anwachsende Kunstliteratur, die europaweit den Kunstdiskurs nicht nur intensivierte, sondern bestimmte Theorien, Kategorien und Termini als positive oder negative Referenzen oder Denkmodelle durchsetzte (Beispiele analysieren Fabian Jonietz und Rebecca Zorach in diesem Band). Die Effekte dieser Prozesse zeigten sich vielfach: Michelangelos Ruhm hätte sich zwar auch ohne Kunstliteratur verbreitet, aber mit Vasaris Vite von 1550 und der dort beschriebenen Stellung in der Entwicklungsgeschichte der Künste war Michelangelo scheinbar endgültig als der Höheund Endpunkt der Arti del Disegno etabliert. Alle jüngeren Künstler hatten sich an diesem Giganten zu messen und zu versuchen, ihm wenigstens in Teilaspekten durch Variation seiner Vorgaben gleichzukommen (zu einem damit zusammenhängenden Phänomen Stephen Campbell): Dies dürfte einer der Hauptimpulse für den Manierismus des 16. Jahrhunderts sein und für die Idee, dass der 'ideale Künstler' die je besten Eigenschaften der großen Meister synkretistisch in sich vereinen müsse. ${ }^{11}$ Mit der Hypothek Vasaris, dann aber auch mit der wenig späteren gegenreformatorischen Kritik an Michelangelo und an der zeitgenössischen Kunstproduktion insgesamt ergab sich die Notwendigkeit einer Neubegründung der Bildkünste. In deren Gefolge wurden Alternativen entwickelt, die nicht nur erstmals zur systematischen Unterscheidung und Charakterisierung von Malerschulen in Italien und Europa führten, sondern auch zu einem nochmals verfeinerten Bewusstsein von historischen Kunststilen und dem künstlerischen Umgang damit sowie schließlich zu einer veränderten Diskussion darüber, wie Neuheit überhaupt in den Künsten erzeugt werden könne. ${ }^{12}$ Alle drei Aspekte: der Wettstreit zwischen unterschiedlichen Kunstzentren, der komplexe Einsatz von verschiedenen Formen alter Kunst

10 Warnke 1985; Campbell 2004; aus den zahlreichen Studien zur Genese des frühneuzeitlichen Kunstmarkts etwa Vermeylen 2003.

11 Vgl. bereits die beiden klassischen Beiträge von Smyth 1992; Shearman 1988; weiterhin etwa Stoichita 1995 und Ames-Lewis/Joannides 2003.

12 Zum Stilbewusstsein Sohm 2001; Hoppe/Müller/Nußbaum 2008; zur Vorstellung vom Wettstreit niederländischer Künstler untereinander und mit der italienischen Kunst Müller, J. 1993; zur Genese des Schul-Begriffs zusammenfassend Pfisterer 2011; zu Neuheit Cropper 2005 und Pfisterer/Wimböck 2011. 
und das gesteigerte Interesse an Neuheit, provozierte wiederum veränderte Formen von aemulatio (dazu Fabian Jonietz und Larry Silver). Insgesamt lässt sich - wie wohl bei jedem Normierungsprozess - beobachten, dass die neuen kunsttheoretischen Kategorisierungen vielfach und vor allem auch von den Künstlern selbst in ihren Werken nur aufgegriffen wurden, um sie entweder möglichst schnell auf die Spitze zu treiben oder gleich wieder in Frage zu stellen, mit den Grenzen zu spielen oder diese ironisch zu brechen - so etwa bei den sich ausdifferenzierenden Gattungsnormen (dazu am Beispiel von Götterdarstellungen Semjon A. Dreiling).

In der Literatur ist die Verbindung von aemulatio und Rivalität weniger spektakulär. Doch auch die Literaten müssen um die Gunst ihres Mäzens wetteifern, wobei literarische Werke häufig als Nebenprodukte professioneller Aufgaben in Rat, Diplomatie, Kanzlei erscheinen, dabei aber gleichwohl für diese empfehlen sollen. Die Festreden, Lobgedichte, mythologischen Spiele, Kasualcarmina zu allen möglichen repräsentativen Anlässen der frühneuzeitlichen Gesellschaft sollen dem Splendor ihrer Adressaten dienen und zugleich ihren Verfassern jene Anerkennung des Gönners verschaffen, die mit der gewöhnlichen Amtstätigkeit nicht zu gewinnen war. Der kaiserliche oder königliche Poet errang zwar tatsächlich nie das Ansehen, den Rang und die Entlohnung eines Hofkünstlers, doch im Bewusstsein der Literaten selbst stand er hinter ihm nicht zurück. ${ }^{13}$ Die Herrscher bemühten sich nicht nur um Propagandisten, die ihre politischen und militärischen Aktionen (zum Beispiel in mündlich vorgetragenen Reden und Liedern) 'verkaufen' mussten, sondern auch um Poeten, deren repräsentative Werke es ihrer Auffassung nach mit Vergil, Horaz oder gar Homer aufnehmen konnten. ${ }^{14}$ Anders als die prunkvollen Herrscherporträts und -standbilder sind freilich die vergilisierenden Epen, die Austrias, Carolis, Franciade usw. heute vergessen, und die unzähligen Festund Huldigungsspiele beschäftigen allenfalls noch die Philologen. ${ }^{15}$

Auch auf diesem Gebiet konnte die Konkurrenz hart sein. Vor allem im Italien des Quattrocento und im Umkreis der Kurie ist das Verhältnis der Literaten untereinander häufig durch Eifersucht, Intrigen, Herabsetzung der literarischen Qualität des Konkurrenten geprägt. ${ }^{16}$ Berufliche Kon-

13 Vgl. Schirrmeister 2003; Flood 2006 u. 2008.

14 Müller, J.-D. 2009.

15 Zu Maximilian- und anderen Herrscherepen Müller, J.-D. 1982; zu den Festpielen des Celtis Müller, J.-D. 2009; zu Ronsard und Franciade vgl. Bjaï 1997, Csűrös 1999, Ferguson 2003, Quint 1993, Rigolot 1988 und Silver 1961.

16 Zur humanistischen Polemik vgl. Rossi, V. 1992, 147-149 und 245-247; Vismara 1990; Ricci 1974, besonders 411-414; vgl. auch Campanelli 2001. 
kurrenz konnte als ästhetischer Wettstreit ausgetragen werden. Debatten wie die um das beste Latein, die beste Nachahmung der Alten, die Geltung einzelner Vorbilder werden mit Erbitterung zwischen verschiedenen Fraktionen geführt. ${ }^{17} \mathrm{Im}$ Wettstreit können einzelne Züge der bewunderten Vorbilder auf Kosten anderer hypertrophiert werden, so dass eine 'Manier' zum Manierismus gesteigert wird, wenn es auch in der Literatur nicht zur Schulbildung wie in der Malerei kommt. ${ }^{18}$

Das aemulative Prinzip wirkt vornehmlich in der Auseinandersetzung mit der Antike, doch dringt es von dort in andere Bereiche: in die Auseinandersetzung volkssprachiger Literaturen untereinander und mit der klassischen, in den Vergleich kultureller Phänomene insgesamt, in das Messen von Status und Repräsentation.

\section{Imitatio/ aemulatio veterum - imitatio naturae}

In Dichtung und bildender Kunst liegen die Verhältnisse also nicht völlig parallel, doch gibt es enge Verwandtschaften. Bedingung der aemulatio ist immer eine anerkannte Autorität, an der man sich misst und welche die Kriterien des Wettstreits definiert. In der Literatur ist das die Autorität der Alten, in den Künsten insgesamt (auch den angewandten) die Autorität von Meisterschaft. Durch aemulatio wird freilich Autorität immer auch in Frage gestellt, die Neuheit (novità) kann auf Kosten des maßstäblichen Alten favorisiert werden, man kann den Kanon unterlaufen und einen Gegenkanon etablieren (weshalb aemulatio auch subversiv wirken kann). ${ }^{19}$

Der literarische Humanismus sucht eine vergangene Epoche als Leitbild des Lebens, Denkens und Schreibens, in Abgrenzung zur 'Barbarei' der jüngst vergangenen Jahrhunderte zu erneuern und damit einen jahrhundertelangen Verlust zu kompensieren. Er entdeckt die antike Philosophie, Geschichtsschreibung, Dichtung als Maßstab für Leben, Denken und Gesellschaft, vor allem aber für die Sprache. Ihnen hat sich der Gebildete imitativ wieder anzunähern. Die klassische Beredsamkeit soll nicht in sklavischer Nachahmung, sondern im freien Wettbewerb adaptiert werden.

17 Müller, J.-D. 1999.

18 Zymner 1995 u. 1997.

19 Müller, J.-D. 2007a. 
Imitatio und aemulatio, die Leitbegriffe der Debatte darüber, ${ }^{20}$ sind auf ein verbindliches Modell des Nachzuahmenden bezogen. Dazu müssen die bewunderten Vorbilder zunächst wiederentdeckt, von mittelalterlicher Entstellung befreit und in Werken, die sich an ihnen orientieren, erneuert werden. Es gilt zunächst, das bewunderte Vorbild zu erreichen. Dies geschieht durch Nachahmung (imitatio).

Die humanistische Wiederbelebung des Begriffsfeldes aemulatio, aemulus, aemulari erfolgte in den Jahrzehnten um und nach 1400 in engstem Zusammenhang mit den Künsten - und nicht erst, wie oft behauptet, knapp ein Jahrhundert später mit Polizian und Erasmus im Zusammenhang des Ciceronianismus-Streits. ${ }^{21}$ Pier Paolo Vergerio definierte in seinem Erziehungstraktat von 1402/1403 geradezu das Wesen und die fundamentale Wirkung von Bildwerken darüber, dass sie zur wetteifernden Nachahmung mit den dargestellten Vorbildern auffordern: „imagines ad aemulationem excitant ". 22 Der Begriff wird hier nicht als rhetorischer oder poetologischer Spezialterminus aufgegriffen, sondern soll in erweitertem letztlich auf die Theorien des Aristoteles zu mimesis und zelos zurückgehendem - Verständnis das Verhältnis von (Bild-)Fiktion und einer Realität charakterisieren, in der die Betrachter sich am Ideal des Bildes messen, verbessern und daraus wettstreitenden Ansporn ziehen. Insofern ist imitatio nicht ein kunsttheoretischer oder poetologischer Spezialbegriff, sondern hat eine umfassend anthropologische Bedeutung: Man soll nicht nur sein Bild, seine Statue, seine Rede oder sein poetisches Werk am klassischen Modell ausrichten, sondern den 'ganzen Menschen' nach dem Vorbild modellieren. ${ }^{23}$

Problemlos werden im Zeichen des Prinzips ut pictura poesis auch die spezifisch rhetorischen und dichtungstheoretischen Kategorien und Exempla auf die Bildkünste übertragen: ${ }^{24}$ Bereits Villani hatte Giotto attestiert, er sei ein „poesis emulator“ ${ }^{25}$ Vorsichtig unterschied 1433 Carlo Marsuppini für Malerei, Skulptur und Architektur zwischen dem Versuch des Nachahmens der antiken Vorbilder und dem Wettstreit mit diesen und

20 Neben den zusammenfassenden Artikeln von Bauer 1992 und Kaminski 1995 auch Gmelin 1932; Cave 1979; Pigman 1980, Greene 1982 sowie Fera 1999 und Fera 2004.

21 Bauer 1992, 164-168; aus kunsthistorischer Perspektive in diesem Sinne etwa Pochat 1987.

22 Vergerio 1917-1918, 103.

23. Müller, J.-D. 2007b.

24 Zur kontroversen Diskussion in den Bildkünsten, an welchen Vorbildern man sich zu orientieren habe, Baxandall 1971; Bolland 1996 und Irle 1997.

25 Villani $1997,154$. 
verdeutlichte so, dass ihm die in der Antike nicht systematisch und theoretisch entwickelte Steigerung von imitatio zu aemulatio bewusst war:

Wenn die Maler, Bildhauer und schließlich Architekten unserer Zeit den Tempeln, Foren, Säulen, Bildern, wenn sie schließlich den marmornen und bronzenen Statuen der Antike auch nur in einem Teil gleichzukommen [aemulari] verstehen, erwerben sie sich höchsten Ruhm [...]. Was zweifeln wir also [noch prinzipiell] daran, die Alten, denen wir laut Servius von ferne folgen und deren Spuren wir verehren, nachzuahmen [imitari] $?^{26}$

Angelo Decembrio kommt in seinem 1462/1463 vollendeten Dialog zur literarischen Verfeinerung, der angeblich Gespräche am Ferrareser Hof bereits der 1440er-Jahre wiedergibt, auf die beiden antiken Skulpturen der Rossebändiger in Rom zu sprechen, die man in der Renaissance als Konkurrenzwerke der Großmeister Phidias und Polyklet (fehl-)deutete. ${ }^{27}$ Damit stellt Decembrio nicht nur seine souveräne Kenntnis der Naturalis historia des Plinius unter Beweis: Denn bereits bei Plinius erscheint der Wettstreit als ein Leitmotiv und Hauptmovens des künstlerischen Fortschritts der griechischen Maler und Bildhauer. Zeuxis maß sich mit Parrhasios darin, wer die feinste Linie ziehen könne. Vor allem aber trugen die Bildhauer Phidias, Polyklet, Kresilas und Kydon - die im Übrigen zu unterschiedlichen Zeiten lebten - einen 'Kampf' (certamen) darüber aus, wer die schönste Amazonenstatue für das Heiligtum der Artemis zu Ephesos erschaffen könne $(34,53)$. Und gerade für die Darstellung von Pferden überliefert der Polyhistor $(34,71)$, dass Kalamis darin 'ohne Konkurrent' („sine aemulo“) gewesen sei. Vor diesem Hintergrund merkt Decembrio nun an, dass nicht nur die beiden antiken Bildhauer mit den beiden Statuen der Rossebändiger sich gegenseitig ihre Kunst beweisen und diese im unmittelbaren Vergleich zugleich verbessern wollten, sondern dass dadurch auch die Renaissancekünstler überhaupt zum antikischen Prinzip des Wettstreits („mutua [...] aemulatione“) angeregt würden. ${ }^{28}$ Ganz entsprechend spielt der Wettstreit mit den antiken wie zeitgenössischen Meistern in der frühen humanistischen Kunstliteratur nördlich der Alpen

26 Marsuppini 1940, 395-397: „An nostri temporis pictores, sculptores atque denique architecti, si templa, si fores, si columnas, si pictas tabulas, si denique marmorea signa statuasque aeneas antiquorum parte aliqua aemulari possunt maximae gloriae sibi ducent, [...] nos antiquos quos, ut inquit Statius, longe sequimur et vestigia semper adoramus, imitari dubitamus?"

27 Vgl. etwa Thielemann 1996; Pfisterer 1999; dazu auch - nicht immer ganz zutreffend - Koch 2004.

28 Decembrio 2002, 426 f. (lib. 6, cap. 68): „Ipsi vicissim artifices opera sua demonstrantes emendabant. Quos nunc mutua novimus aemulatione lacessiri“. 
eine zentrale Rolle - etwa in Christoph Scheurls beiden Lobeshymnen auf Dürer und Cranach (1508/1509). Alle diese Aspekte - insbesondere auch die Vorstellung vom paragone der Künste - finden sich dann etwa in Charles-Alphonse Dufresnoys einflussreichem Bildgedicht De arte graphica (1668) zusammen geführt, wo es gleich im ersten Satz heisst: „Ut Pictura Poesis erit, similisque Poesi / Sit Pictura; refert par aemula quaeque sororem, / Alternantque uices et nomina; [... “.29

Und auch in der künstlerischen Praxis zeigt sich die Wirksamkeit dieser Ideen: So sprechen eine ganze Reihe von Indizien dafür, dass etwa Donatello in den 1430er- und frühen 1440er-Jahren sein Verhältnis zu den antiken Vorbildern - und insbesondere zum vermeintlich besten antiken Bildhauer Polyklet - von einem imitativen zu einem aemulativen steigerte, möglicherweise sogar bis hin zum Bewusstsein einer Überwindung ( $s u$ peratio) des antik-heidnischen Künstlers durch den christlich-modernen. ${ }^{30}$ Michelangelos täuschende Antikennachahmungen, der kontinuierliche Wettkampf zwischen Michelangelo und Raffael (beziehungsweise ihren jeweiligen Anhängern), die Konkurrenzen von Baccio Bandinelli und Benvenuto Cellini mit der Antike und mit Michelangelo, oder Hans Baldung Griens lebenslange Auseinandersetzung mit dem Vorbild Dürer die Reihe ließe sich fast beliebig fortsetzen - führen unterschiedliche Formen eines große Teile ihres Werkes bestimmenden ästhetischen Wettstreits vor. ${ }^{31}$

In der vielleicht prominentesten rhetorik- und literaturtheoretischen Debatte des 15. und 16. Jahrhunderts, dem Ciceronianismus-Streit, behauptet nur die eine Seite die Unüberbietbarkeit der Antike Ciceros; die andere empfiehlt mehrere Muster, aber auch dabei geht es zunächst um angemessene imitatio, nicht aemulatio. ${ }^{32}$ In Erasmus' Ciceronianus fürchtet Nosoponus, der sterile Ciceronianer, wie die Pest, in irgendeiner Hinsicht anders zu reden als Cicero. Deshalb beschränkt er nicht nur sein Vokabular auf Wörter, die auch Cicero schon gebraucht hat, sondern sogar ausschließlich auf von Cicero gebrauchte Wortformen, schließt dagegen jede grammatische Abweichung vom Corpus Ciceronianum aus, würde er damit

29 Dufresnoy 2005, 178 ('Dichtung sei wie Malerei, und Malerei sei wie Dichtung; sie sind Schwestern und Rivalinnen; sie tauschen ihre Rollen und Namen; [... $]^{\prime}$ ).

30 Pfisterer 2002.

31. Barbieri 2005, zu Baldung und Dürer Koerner 1993; vgl. jetzt auch Cole 2011.

32 Vgl. hierzu die Debatte zwischen Paolo Cortese und Angelo Poliziano (Cortese / Poliziano 1952) sowie Gianfrancesco Pico de la Mirandola und Pietro Bembo (Pico/Bembo 1954). 
doch das bewunderte Vorbild verfehlen. ${ }^{33}$ Gewiss ist dieser Ciceronianer eine Karikatur, an der ein angemessenerer Umgang mit der antiken Tradition profiliert werden soll, doch ist die Position der Anti-Ciceronianer grundsätzlich gar nicht so verschieden. Sie plädieren ja dafür, aus verschiedenen Meistern der lateinischen Sprache sich die passenden Vorbilder auszuwählen, und zwar nach Maßgabe der Individualität der Auswählenden. Statt zur simia Ciceronis solle sich jeder Redner oder Poet zu der Vollkommenheit bilden, die ihm, seinen Umständen und seiner Zeit angemessen ist. Wer auf seine Weise, mit Hilfe der ihm adäquaten Vorbilder und unter den Umständen, unter denen er spricht, seine Rede vervollkommnet, der hat die bewunderten Alten erreicht. Solange das klassizistische Stilideal herrscht, ist deshalb imitatio als poetologisches Prinzip wichtiger als aemulatio.

Allerdings sind Zeit und Umstände verändert: Man lebt in einer christlichen Welt, die als solche der heidnischen immer überlegen ist, und unter gänzlich anderen politischen Umständen. Schon der Imitatio-Debatte ist also das Bewusstsein kultureller Differenz eingeschrieben, deutlicher bei den Gegnern des Ciceronianismus. Individualität und Geschichtlichkeit als die Prinzipien, die man mit fortschreitender Nachahmungsdebatte entdeckt, sind Kategorien der Andersheit, die der Angleichung Grenzen setzt. Antike Gattungsmodelle werden mit christlichen Stoffen gefüllt, so dass die neuen Werke ihre Vorbilder übertreffen. In der Literatur gewinnt deshalb seit Mitte des 16. Jahrhunderts der Wettstreit mittels Erfindung des Neuen, immer stärkeres Gewicht (an Naogeorgs Bibeldrama zeigt dies Regina Toepfer).

Bildkünste wie Dichtung werden auf möglichst lebensechte und naturnahe Darstellung verpflichtet. Imitatio/ aemulatio veterum und imitatio naturae werden in eins gesehen. Maler, Bildhauer und Dichter der Renaissance ahmen die Natur nicht nur nach, sie wetteifern zunehmend mit ihr darin, dass die von Menschen geschaffene zweite Natur entweder die Sinne vollkommen zu täuschen vermag oder aber noch besser und vollkommener als die natura naturata erscheint (allein der 'Wettstreit mit Gott' scheint zunächst ein Tabu - hierzu die Überlegungen bei Bremer). Seit sie durch Giotto erneuert wurde, ist die Malerei - so Filippo Villani bereits in den 1380er-Jahren - „emulatri[x] nature“. ${ }^{34}$ Die im Laufe des 15. und 16. Jahrhunderts zunehmend thematisierte Spannung zwischen möglichst genauer Naturnachahmung und die Wirklichkeit übersteigender Ideali-

33 Erasmus 1972; vgl. Pigman 1980; Müller, J.-D. 1999.

34 Villani 1997, 155; vgl. dazu Baxandall 1971, 71 und 147. 
sierung - zwei unterschiedliche Formen des Wettstreits mit der Realität führten dazu, dass dann um 1600 die Wendung von Kunst oder Künstler als „Wettstreiter mit der Natur" vielfach positiv wie negativ verwendet und ausgedeutet werden konnte. Das eine Extrem markiert Federico Zuccari, dem die ideale geistige Konzeption, die geradezu als metaphysisches Leitprinzip verstandene 'innere Zeichnung' (disegno interno) erscheint als "quasi un'altra natura generante, com'emulo della natura viva. “35 Dagegen hörten sicherlich viele Zeitgenossen bei der Charakterisierung Caravaggios als „naturae aemulator" negative Untertöne über dessen bedingungslosen Naturalismus mitschwingen. ${ }^{36}$

Besonders interessant ist dabei die Schnittstelle zwischen ästhetischem und instrumentellem Handeln. In dem Maße, in dem in der Kunst die mühevolle Anstrengung (labor) als eine Bedingung erfolgreicher aemulatio bedacht wird (dazu die Ausführungen von Fabian Jonietz), gerät labor in ganz anderen Bereichen unter den Einfluss des aemulatio-Prinzips. Auch die Anstrengung instrumentellen Handelns kann als Wettstreit, Wettstreit nämlich mit der Natur, begriffen werden (vgl. die Beispiele bei Martin Schmeisser). In diesem Wettstreit geht es nicht mehr um das vollkommene Kunstwerk, sondern um die Herrschaft des Menschen über die Natur, letztlich um Wissenschaft und Technik, die sich in der Folge aus diesem Zusammenhang lösen werden. Auch hier gibt es den Wettstreit mit dem Konkurrenten. Als Extremfall epochenspezifischer aemulatio kann deshalb sogar die rekordverdächtige Nomenklatur, mit der sich Leonhard Thurneysser als Gelehrter in Szene zu setzen sucht, betrachtet werden (ihm widmet sich Tobias Bulang).

\section{Grenzen der imitatio}

Die imitatio veterum stößt überall dort an Grenzen, wo antike Vorbilder fehlen oder mindestens nicht überliefert sind. Wenn sie fehlen, kann Kunst nicht imitativ produziert werden. Wenn Vorbilder im Kunstwerk hingegen nicht markiert sind (Barbara Mahlmann-Bauer) oder verloren gegangen

35 Zuccari 1961, 34 (Paginierung 1607). Vgl. etwa Demontiosius 1585, 1: „Fictorum vero duo sunt genera. Unum eorum qui signa faciunt, quae quaquaversus spectentur naturae opus aemulantur. [...]“".

36. So das Epitaph in den Inscriptiones et Elogia des Marzio Milesi, Biblioteca Apostolica Vaticana, Cod. Vat. 7927, 133 ${ }^{\mathrm{r}}$. Ein anderes Gedicht Milesis spricht von der „naturae aemulatione“. Vgl. Macioce 2003, 264 (II DOC 403 und 404). Zu den Diskussionen etwa Dempsey 1993; Brown 2001; Moffitt 2004. 
sind (Kai Bremer), können imitative Verfahren nicht beurteilt werden. Dies betrifft bildende Kunst wie Literatur.

Für die Bildkünste führte die unterschiedliche Überlieferungssituation dazu, dass man in Italien der Antike bei der Bildhauerkunst angesichts zahlreicher erhaltener Beispiele zunächst den Vorrang zugestand, wogegen sich bei der Malerei, für die alle antiken Vergleichsstücke verloren schienen, leichter behaupten ließ, dass hier einige moderne Maler den griechischen Vorläufern zumindest gleich kämen, wenn sie diese nicht sogar aufgrund der Kenntnis der Zentralperspektive und anderem überträfen. ${ }^{37}$ Nördlich der Alpen präsentiert sich die Situation wohl noch eine Stufe komplizierter: Die antigisch art, worunter zunächst Antike wie italienische Renaissance verstanden wurden (erst im Laufe des zweiten Viertels des 16. Jahrhunderts wurde begrifflich zwischen Antike und Renaissance zu differenzieren begonnen), scheint hier zumindest von einigen Künstlern und Kunstliteraten von Anfang an in ihrem vorbildlichen Wert kritisch beurteilt worden zu sein. Es ist daher zukünftig noch intensiver zu fragen, ob vermeintliche Antikennachahmung und Wettstreit insbesondere nördlich der Alpen nicht vielmehr Kritik oder sogar eine radikale Alternative zum Modell Antike / Italien implizieren. ${ }^{38}$ Noch weiter geht ein aktueller Vorschlag, der überhaupt eine vom humanistischen Italien und der Moderne grundsätzlich abweichende Zeitvorstellung und damit ein ganz anderes Wahrnehmen von Abfolge und Präsenz von Kunstobjekten postuliert. ${ }^{39}$

In der Literatur stellt sich das Problem anders: Die unterschiedlichen Strukturen und Traditionen lateinischer und vernakularer Texte lassen Nachahmung nur auf bestimmten Ebenen zu. Deshalb sieht die Auseinandersetzung mit der Antike innerhalb der neolateinischen Literatur anders aus als in volkssprachigen Texten und hier noch einmal anders in den romanischen Volkssprachen als in denen mit germanischer Wurzel.

37 Decembrio 2002, 428: „Alios contra et quidem paucos uideas antiquitatis pictores aemulos: qui exquisitissime omnem prius corporis staturam quam effingere volunt, ita remetiuntur: ut membra membro nullum propius longiusve consistat [...]" ("Einige wenige andere wiederum - wie du siehst - wettstreiten mit den Malern der Antike: Zuerst ermitteln sie sorgfältigst alle Maße des Körpers, den sie darstellen wollen, dergestalt, dass kein Körperglied zu kurz oder lang im Verhältnis zu einem anderen ist [...]'). Dazu auch Baxandall 1963. Zur unterschiedlichen Bewertung von Bildhauern und Malern im Vergleich zur Antike bereits Francesco Petrarca, Rerum familiarum libri, V, 17 und Giovanni Dondi (vgl. Gilbert 1977, 336 f.). Zur weiteren Auseinandersetzung Joyce 1992 und Dundas 1998.

38 Müller, J. 2007; Weissert 2008; Kaschek 2009; Metzger 2010.

39 Nagel/Wood 2010; vgl. dazu die Besprechung von Fehrenbach 2011. 
Das germanistische Projekt innerhalb des SFB war zunächst der imitatio veterum gewidmet gewesen. Es stellte sich jedoch heraus, dass in Bezug auf volksssprachige Literatur diese als poetologisches Prinzip vor Martin Opitz' Deutscher Poeterey ${ }^{40}$ immer nur defizitär gelingt. Sie kann im Verhältnis zu den lateinischen und griechischen Klassikern nur in engen Grenzen (zum Beispiel in Bezug auf Stoffe oder Motive) imitativ sein. Ein instruktives Beispiel ist Naogeorgs Tragödie Hamannus. Der neolateinische Autor konkurriert noch mit antiken Tragödien- und Komödiendichtern durch die novitas seiner Poetik. In der Übersetzung geht dieser Aspekt verloren. Der Übersetzer begnügt sich im Wesentlichen mit einer bloßen Wiedergabe der Inhalte (Regina Toepfer).

Diese Beobachtung ist symptomatisch. Sie hat das Bild der deutschen Literatur im 16. Jahrhundert durchweg negativ beeinflusst: Diese wird als 'grobianisch' und roh betrachtet, bestenfalls als ungeschickte Vorgeschichte der 'eigentlichen', klassizistisch geprägten Nationalliteratur seit dem Beginn des 17. Jahrhunderts. ${ }^{41}$ Es ist dies das Bild, das schon Opitz selbst und der Heidelberger Kreis um Zincgref erzeugen und das bis heute die Literaturgeschichtsschreibung bestimmt. ${ }^{42}$ Dabei gibt es im 16. Jahrhundert durchaus das Bemühen, den bewunderten Alten etwas Gleichwertiges an die Seite zu setzen. Dies kann schon von der Struktur der Sprachen her, ihrer Prosodie, der darauf bezogenen Metrik, der Bildtraditionen usw. nie imitativ geschehen, sondern nur aemulativ: indem man den vorbildlichen Modellen etwas Eigenes entgegensetzt. Autoren wie Johann Fischart haben die Reflexion über kulturelle Differenz zum Ausgangspunkt einer manieristischen Überbietungspraxis genommen. ${ }^{43}$ Doch steht Fischart weniger isoliert da als meist angenommen. Auch Texte, die scheinbar weit vom klassischen Kanon entfernt sind, sind nicht einfach als zurückgeblieben, als vorliterarisch-volkstümlich (so die häufige Etikettierung) aufzufassen, sondern bemühen sich um eigene poetologische Prinzipien, die weniger als Verfehlung klassischer Normen denn (wie rudimentär auch immer) als deren Alternative verstanden werden wollen. ${ }^{44}$

$40 \mathrm{Vgl}$. Nachwort und Anmerkungen von Herbert Jaumann in Opitz 2002.

41 Müller, J.-D. 2011.

42 Vgl. das Vorwort von Braune in Zincgref 1879; zur Rolle Zincgrefs jetzt Kühlmann 2011.

43 Müller, J.-D. 2007a.

44. Robert 2007; Bleuler 2009; zum Verhältnis von volkssprachiger Literatur und Humanismus auch: Kühlmann/Neuber 1994 und - besonders wichtig zu Zincgref - Kühlmann 2011; Drücke 2001; Grenzmann/Grubmüller 2004; Plank 2004; Küenzlen 2005; Bußmann 2005; Föcking/Müller, G. M. 2007. 
So spielt das Prinzip der aemulatio zwar von Anfang an in der humanistischen Debatte einer Auseinandersetzung mit der antiken Literatur eine Rolle, aber es dominiert innerhalb der neolateinischen Literatur doch erst im späthumanistischen Manierismus, während es in der volkssprachigen Literatur, überall dort, wo es keine gemeinsame Basis gibt, von Anfang an im Vordergrund steht. Das fängt schon mit Petrarcas Canzoniere an, der ohne antikes Vorbild noch als geringerwertig gilt, und bestimmt durchweg das Verhältnis der lateinischen zu den volkssprachigen Literaturen. Auch bei den volkssprachigen Autoren ist meist vorauszusetzen, dass sie eine Lateinschule besucht haben und mit den poetologischen Diskussionen in der Gelehrtensprache wenigstens ansatzweise vertraut waren. Jedenfalls zeigt sich, dass das Prinzip der aemulatio, wie es etwa bei Quintilian erscheint, auch in der impliziten Poetik volkssprachiger Texte nachweisen lässt (vgl. den Beitrag von Barbara Mahlmann-Bauer). Wo Unvergleichbares in Wettstreit gibt, kann es nicht um ein besonders hohes $\mathrm{Maß}$ an Übereinstimmung geben, sondern nur um Abwägen der Leistung gegeneinander.

Es mag unter diesem Aspekt nicht mehr überraschen, dass nicht weniger als vier Beiträge zum frühneuzeitlichen Roman aufgenommen wurden, zu einer Gattung also, die erst zweihundert Jahre später und mit erheblichen Anstrengungen in den Kanon der anspruchsvollen Literatur integriert wurde, nachdem sie zuvor als subliterarisches Genre zwar erfolgreich, aber allseits geschmäht war. In einer an der Antike orientierten Renaissancepoetik kommt der Roman nicht vor, doch sind die rudimentären Ansätze einer Romanpoetik von ihr nicht unbeeinflusst. Scaligers Umwertung des Wettstreits zwischen Homer und Vergil eröffnet auch zeitgenössischen und selbst volkssprachigen Texten Möglichkeiten aemulativen Vergleichs (Mahlmann-Bauer). Die Kriterien für das Mehr oder Weniger, für das Besser oder Schlechter sind meist nur indirekt zu erschließen, etwa wo die Neuheit eines Werks oder seine Einrichtung nach dem Ordo naturalis betont werden, die dem Leser zusammen mit dem Verzicht auf auktoriale Diegese neue Spielräume der Interpretation eröffnet (Jan Hon). Im Zeichen der aemulatio kann die Neubewertung der Gegenstände stehen (etwa im Verhältnis protestantischer Ehemoral zur erotischen Lizenz im angeblich uralten antiken Muster) und - abseits humanistischer Kunsttheorie - im paragone von Dichtung und bildender Kunst (Sylvia Brockstieger). ${ }^{45}$ Serielles Erzählen ist auf Überbietung an-

45 Die Dominanz des humanistischen Paradigmas zeigt sich darin, dass der mittelalterlich-byzantinische Text zum uralten antiken Erbe umdefiniert wird, die 
gelegt, und selbst ein von klassischen Mustern denkbar weit entfernter Typus verschraubter Rede kann zum Distinktionsmerkmal werden (Henrike Schaffert).

\section{Wettstreit der Kulturen}

Wenn eingangs die These aufgestellt wurde, dass der Wettstreit der Bildkünste möglicherweise als Leitmodell auch für andere Bereiche der Kultur dienen konnte, so zeigt sich dies neben der herausgehobenen Stellung des Disegno $^{46}$ wohl am deutlichsten bei den Theorien und Modellen zur kulturellen Entwicklung insgesamt. Aemulatio hat ja zwei Zeitdimensionen, eine synchrone - man tut sich gegenüber Zeitgenossen hervor - und eine diachrone - man misst sich an der Antike. Es ist der erste Aspekt, der hier zunächst im Vordergrund steht. Von besonderem Interesse für Deutschland sind dabei die Fälle, in denen gelehrte und volkssprachige Werke in Konkurrenz treten: wo eine deutsche Hofkultur sich an anderen vernakularen Hofkulturen misst (Anna Kathrin Bleuler), wo heimische Schwanküberlieferung Stichwortgeber für neolateinische Poesie ist (Wilhelm Kühlmann), wo nord- und südeuropäische Kunstformen in Wettstreit treten (Jörg Robert), wo antikes Traditionswissen durch eine entfesselte Ansammlung von Wissensbeständen überboten werden soll (Tobias Bulang).

Im Wettstreit der Künste um den Vorrang der Dichtung oder der Malerei, der bis zu Lessings Laokoon ein Lieblingsthema ästhetischer Diskurse ist, in der Erörterung der malerischen Qualitäten von Dichtung und der dichterischen Qualitäten von Malerei, im paragone der Bildkünste untereinander, ${ }^{47}$ in der Diskussion um energeia und enargeia liegt die Bedeutung von aemulatio auf der Hand, aber sie ist nicht geringer auf anderen Feldern der Kultur. Eine Rede zum Beispiel kann sachdienlicher als eine andere sein und sich deshalb im Meinungskampf gegen diese durchsetzen. Sie kann insofern unter dem Aspekt ihres praktischen Erfolgs betrachtet werden. Dieselbe Rede wetteifert aber auch mit anderen unter dem Gesichtspunkt kunstvoller elocutio, der gekonnten Adaptation antiker Muster, der Gewandtheit des Vortrags (Claudia Märtl). Beides ist nicht deckungsgleich. Manche der exquisitesten rhetorischen Kunststücke der

Ausstrahlung der aemulatio über den humanistischen Rahmen hinaus, dass man sich für den denkbar unklassischen Text interessiert.

46 Williams 1997.

47 Zusammenfassungen dazu von Pfisterer 2003; Mai/Wettengl 2002; Baader / Müller Hofstede 2007. 
Frühen Neuzeit wirkten auf zeitgenössische wie spätere Rezipienten im pragmatischen Zusammenhang als öde Exerzitien, ${ }^{48}$ akademische Spielereien, die nichts mehr mit dem äußeren und inneren aptum antiker Redepraxis zu tun hätten, ${ }^{49}$ doch den Kennern rhetorischer Finessen bereiteten stilistische Eleganz, Gesuchtheit der Gedanken und virtuose Verwendung von Tropen höchsten ästhetischen Genuss.

In diesem Sinne ist auch das Turnier nicht nur als sportlicher Wettkampf zu betrachten und erst recht nicht als Surrogat ernsthafter Kämpfe um militärischen, politischen oder wirtschaftlichen Vorteil. ${ }^{50}$ Es geht um einen Wettstreit 'schöner' Selbstdarstellung, aristokratischer Vorzüge und Fertigkeiten in einem 'schönen' Rahmen. Aus diesem Grund gewinnen in der Frühen Neuzeit neben den sportlichen Leistungen der Glanz der Ausstattung und die Eleganz des Auftretens immer größere Bedeutung (Claudius Sittig). Das Turnier ist Spiel und als Spiel den elaborierteren Spielen der Dichtkunst oder der Bildenden Kunst verwandt. Daher enthalten frühneuzeitliche Turniere häufig auch theatrale Komponenten und stehen im Zusammenhang anderer höfischer rappresentazioni. ${ }^{51}$ Der Übergang zum Theater ist fließend. Und wie das Turnier sind auch Feste und Zeremonien (Entrées royales, Krönungen, Hochzeiten, Geburten, Geburtstagsfeste, Beerdigungen usw.) Gegenstand des Wettstreits.

Die frühneuzeitliche Kultur ist auf demonstrative Prachtentfaltung oder magnificentia angelegt, die seit dem 14. Jahrhundert wieder durch antike Texte gerechtfertigt wird. ${ }^{52}$ Hier zeigt sich besonders spektakulär, wie aemulatio Kunst- und Lebensformen übergreift. Die komplizierten Ebenen dieser Überbietungslogiken und ihrer politischen, gesellschaftlichen und moralischen Rechtfertigung kann dabei etwa der Wiener Fürstenkongress von 1515 mit dem Kaiser und den Königen von Polen und Ungarn oder die Begegnung des französischen und englischen Königs,

48 Wie es bei den Redeturnieren anlässlich höfisch-politischer Ereignisse tatsächlich zuging und wie sich gerade vornehme Adressaten dabei langweilten, schildert sehr anschaulich der italienische Humanist Ricardo Bartolini (Vgl. Bartolini 1515, B 2 ${ }^{\text {; }}$; $\mathrm{M} 4^{\mathrm{r}}$ ).

49 So macht Erasmus 1972 im Ciceronianus darauf aufmerksam, dass die politischen Reden der Ciceronianer unter den völlig veränderten politischen Verhältnissen leeres Wortgeklingel seien.

50 Dass man Turniere kämpft, um von den unterlegenen Gegnern Lösegeld zu erpressen, gibt es im Spätmittelalter auch.

51 Müller, J.-D. [im Druck].

52 Green 1990. Aus der umfangreichen Literatur zur Kategorie der magnificentia in der Frühen Neuzeit sei nur auf Goldthwaite 1996 verwiesen. 
François I. und Henry VIII., auf dem 'Güldenen Feld' und die Zweikampfherausforderungen des Franzosen an den deutsch-römischen Kaiser 1526/1528 und 1536 liefern. Herrschafts-Konkurrenz wird hier zu einem ästhetischen Wettstreit, realer Kampf zu Zermoniell, Turnier, Kunst und Theater 'sublimiert'. ${ }^{53}$ Dies setzt sich auf scheinbar ganz anders gelagerten Ebenen fort, so in der beliebten, im 17. Jahrhundert etablierten Form der Galerie-Hängung mit Gemälden dicht an dicht gereiht, als Ausdruck herrschaftlicher, auch schon teils großbürgerlicher Prachtentfaltung. Nicht nur verlangte die schiere Fülle dieser Bilderwände, dass die einzelnen Werke nun um die Aufmerksamkeit der Betrachter konkurrierten. ${ }^{54}$ Die in den Sammlungen nachträglich hergestellten Nachbarschaften ergaben Gegenüberstellungen mit den anderen Meistern der Vergangenheit und Gegenwart aus ganz Europa (so wie bereits bei den Amazonen im Heiligtum der Artemis Ephesia). Und noch darüber hinaus und auch außerhalb dieser Galerien verschärfte sich die Vergleichssituation: Vor allem durch die Druckgraphik wurden die besten Werke überall schnell verfügbar und als Kanon etabliert.

Doch der Wettstreit erfasst auch ganz andere Bereiche, etwa den Vergleich der verschiedenen Sprachen. Er wird nicht um die Frage ausgetragen, welche Sprache zur Bewältigung von Alltagsproblemen am geeignetsten ist, und es wird nicht einmal nur gefragt, welche Sprache dank ihrem terminologischen Ausbau am besten zur Diskussion philosophischer oder sonstiger wissenschaftlicher Probleme taugt, sondern man erörtert, welche Sprache besser als andere Dichtung, Redekunst und gebildete Konversation auszudrücken in der Lage ist. ${ }^{55}$ Damit ist freilich nicht ein poetischer Spezialdiskurs gemeint, sondern eine elaborierte Rede, über welche die verschiedenen sozialen Eliten auf je eigene Weise verfügen: der Hofmann, der Gelehrte, der fürstliche oder städtische Amtsträger.

Die Volkssprachen sind besonders attraktive Felder der aemulatio, des Wettstreits mit den Sprachen der klassischen Antike wie des Wettstreits untereinander (Anna Kathrin Bleuler). Nirgends sind die Möglichkeiten aemulativen Sprechens größer. Auch in Deutschland beruft man sich auf Bembo und seinen Versuch, in Italien das Toskanische der tre corone Dante,

53 Dazu ausführlich Tauber 2009. Ein anderes gelagerter Fall bei Michalsky 2005.

54 Stoichita 1998; Welzel 2006; Wimböck 2008.

55. Dies verbindet die einschlägigen Traktate und Dialoge Bembos, Baldesar Castigliones, Sperone Speronis, Du Bellays (Müller, J.-D. 1999 u. 2007b); daher die Teilnahme von Vertretern unterschiedlicher sozialer Gruppen an der Diskussion: Die Gelehrten stellten andere Anforderungen an die Sprache als die Hofleute. 
Petrarca und Boccaccio als Leitmedium gegen die lateinische Gelehrensprache durchzusetzen. ${ }^{56}$ Über die Sprachen wetteifern die Nationen miteinander. Gleichrangigkeit oder gar Überlegenheit auf einem Gebiet in Deutschland etwa auf dem der Bibelübersetzung - kann als Argument für Gleichrangigkeit auf einem anderen dienen. ${ }^{57}$ Und wenn die Deutschen in Bezug auf die Musen nicht mit den antiken Poeten konkurrieren können, dann doch in ihrer alt Teutsch Tugendmutsamkeyt. Die tugendhafte Gesinnung, der Entschluss zur Tugend - so könnte man vielleicht übersetzen - steht an der Stelle dessen, was in der Antike die Musen verliehen: „[...] O du Mutsamkeyt!/Die sonst wirdst fur die Musam bschreyt" ${ }^{\text {.58 }}$

Für die bildenden Künste lassen sich zumindest ansatzweise ähnliche Phänomene des 'aemulativen Übersetzens' beobachten: Nicht nur nimmt die Mobilität der Künstler und ihrer Werke ständig zu, so dass unterschiedliche Traditionen und Stile aus ganz Europa an einem Ort konfrontiert werden können (Giorgio Vasari etwa hat diese Zusammenhänge für die nach Florenz und Rom kommenden Künstler beschrieben).$^{59} \mathrm{Da}$ die Künstler zu Ausbildung oder Anstellung zunehmend auch ihre Werke verschickten, entsteht eine neue Form des Wettstreits durch den Kulturvergleich und Kulturtransfer (dazu Jörg Robert und Maurice Saß in diesem Band): In Anlehnungen an literaturtheoretische Diskussionen scheint es auch Versuche gegeben zu haben, einen spezifisch 'volkssprachigen' Malstil auszubilden, im Unterschied zu und in Konkurrenz mit der heroisch-antikischen Formensprache. ${ }^{60}$

56 Fischart 1895, 122 f.: „Daher sagt recht der von Latinischer Wolredenheit berümt Cardinal Bembus inn seinen Italiănischen Prosis, darinn er von vbung vnd erweiterung der gemeynen Landsprach handelt, das die jenigen, welche also grose sorg, fleis vnd müh anwenden auff eyne sprach, die allbereyt erweitert vnd zum herrlichsten auspalirt vnd zum hóchsten gebracht worden, gleich wie die Griechische und Latinische ist, nur holz inn Wald, Sand zum Mór vnnd Wasser inn Bronnen tragen oder, wie wir sprechen, füren Håfen gen Hagenau vnd Ruben gen Straßburg“" (Vorrede Bernhard Jobins).

57 Fischart 1895, 123: „so werden wir erfaren, das Gott, der inn allen Sprachen will gelobt sein, auch inn vnserer Sprach wird wunder wircken, wie er dan allbereyt mit der Theology hat erwisen, das man dieselbige so deitlich, hell vnd reyn als inn andern Sprachen mag lesen; kann er das inn eynem, so kann ers auch inn meherm ".

58 Fischart 1588, V. 79, 85 f.; vgl. Müller, J.-D. 2007a.

59 Clifton 1996; vgl. für die Praxis etwa Bornscheuer 2008.

60 Dies lässt sich sowohl in Italien feststellen, etwa wenn Raffael gegen Antike und Michelangelo opponiert, dazu Reilly 2010, als auch für das Verhältnis der nordalpinen Bildkünste zum vermeintlichen Vorbild der Antike und der italienischen Renaissance. 
Oder es werden 'Blütezeiten' der Nationen verglichen. In seiner Vorrede zu Mathias Holtzwarts Emblematum Tyrocinia misst Johann Fischart das gegenwärtige Deutschland an der „Jonischen/Toscanischen/Corinthischen vnd Römischen Blo̊zeit". "Sinnreiche künst" - Architektur, Kunsthandwerk - blühen heute wie bei den Griechen; "heutigs Tags“ würden sie "nicht alleyn den Alten abgelehrnet", sondern es gebe auch neue, die diese überbieten, es den Alten „nicht so sehr nah/als zu und vorthun"; hinter Meisterwerken heutiger Goldschmiedekunst müssten berühmte antike Beispiele weit „dahinden stehn“, und was die Kriegskunst betrifft, so ist „keyne Nation [...] fleissiger inn dergleichen Kriegszeichen als die Teutschen gewesen ".61

Dieser Wettstreit der Nationen hat nichts mit dem Nationalismus des 19. Jahrhunderts zu tun, sondern er ermöglicht den Kulturvergleich, in dem es darum geht, spezifische Besonderheiten in Abgrenzung zu anderen zur Vollkommenheit auszubilden, Besonderheiten eines regionalen Stils, regionaler Schulbildung und dergleichen Vergleiche, Profilierung des Eigenen durch das Zitat des Fremden, die autoritative, affirmative, subversive, parodierende Anspielung sind insofern nicht nur Motor künstlerischer Produktion, sondern bestimmen alle Felder kultureller Praxis.

\section{Kampf der Akteure und historischer Wandel}

Dies führt am Schluss nochmals zurück zum grundlegend mit aemulatio verbundenen Problem von Bewahrung von Traditionen einerseits, von (Neu-)Erfindung, Fortschritt und Verdrängung andererseits. Denn so sehr die aus Rhetorik und Poetik übernommenen imitatio-Lehren auch die Kulturtheorie der Frühen Neuzeit prägten, die Distanz zur Antike ebenso wie das Fortschreiten der Geschichte waren nicht zu bestreiten. Das begann bereits mit Dantes Feststellung (Purg. 13, 79-99), dass Oderisi aus Gubbio das Feld der Buchmalerei, Cimabue das der Monumentalmalerei zu beherrschen glaubte, nur um von Franco Bolognese und Giotto verdrängt zu werden. ${ }^{62}$ Wenn dann mit Petrarca die Selbstreflexion über die eigene historische Stellung und die Relation zu den vorausgehenden Zeiten des Mittelalters und den fernen, untergegangenen der Antike einsetzte, dann resultierte daraus in neuer Dringlichkeit die Auseinandersetzung von antiqui und moderni über den jeweiligen Stellenwert ihrer Kultur: Auch

61 Holtzwart 2006, $7 \mathrm{f}$. und 13.

62 Zuletzt Burioni 2011, $960 \mathrm{f}$. 
Petrarca rief hierfür den Vergleich von antiken und modernen Bildhauern und Malern auf. ${ }^{63}$

Wettstreit und Konkurrenz werden als Movens aller kulturellen Entwicklungen erkannt - nachdem in der Antike bereits Heraklit den Krieg zum Vater aller Dinge erklärt hatte - so etwa bei Lorenzo Valla 1455. Auch hier spielt das anschauliche und damit beweiskräftige Beispiel der Bildkünste eine wichtige Rolle: „Denn es ist von Natur aus so angelegt, dass nichts richtig fortschreiten und wachsen kann, was nicht von mehreren betrieben, bearbeitet und verbessert wird - insbesondere wenn diese miteinander wetteifern und um das Lob kämpfen. Wer hätte denn als Bildhauer, als Maler etc. in seiner Kunst als vollkommen und groß herausgeragt, wenn er der einzige Künstler seiner Disziplin gewesen wäre? Jeder erfindet etwas anderes, und was jemand bei einem anderen als herausragend erkannt hat, das versucht er selbst nachzuahmen, dem gleichzukommen und es zu übertreffen [imitari, aemulari, superare]. So werden die Studien befeuert, vollzieht sich Fortschritt, wachsen die Künste und gelangen zur Vollendung, und dies umso besser und schneller, je mehr Menschen an ein und derselben Sache arbeiten ". ${ }^{64}$

Mit aemulatio wird die soziale Dimension kultureller Entwicklung angesprochen. Jacob Canters Dyalogus de solitudine, 1491/1492 zwischen Augsburg und Landshut verfasst, entwickelt die angedeutete Theorie in Buchlänge. Gegen die üblichen Lobeshymnen auf die Einsamkeit, den einfachen Zustand der Menschheit und das Goldene Zeitalter argumentiert Canter für die Vorzüge der Gegenwart und das gemeinschaftliche Stadtleben, denn Fortschritt und maximale Verwirklichung der menschlichen Erfindungskräfte können für ihn nicht in Isolierung, sondern überhaupt nur durch gemeinschaftlichen Wettstreit, Konkurrenz und Vergleich stattfinden. Auch dem nordalpinen Humanisten dienen die Künste als Beispiel: „Glaube mir, Zeuxis und Lysippus oder auch die anderen berühmten antiken Maler und Bildhauer hätten nie diesen Hö-

63 Dazu Donato 2003. Zum 15. und frühen 16. Jahrhundert Trinkaus 1987. Vgl. dann etwa das Beispiel bei Herrmann-Fiore 1996.

64 Valla 1962, 282: „Namque ita natura comparatum est, ut nihil admodum proficere atque excrescere queat, quod non a plurimis componitur, elaboratur, excolitur, praecipue aemulantibus invicem et de laude certantibus. Quis enim faber statuarius, pictor item et ceteri, in suo artificio perfectus aut etiam magnus extitisset, si solus opifex eius artificii fuisset? Alius aliud invenit, et quod quisque in altero egregium animadvertit, id ipse imitari, aemulari,superare conatur. Ita studia incenduntur, profectus fiunt, artes excrescunt et in summum evadunt, et eo quidem melius eoque celerius, quo plures in eandem rem homines elaborant". 
hepunkt in ihrer jeweiligen Kunst erreicht, wenn sie in Einsamkeit und in den Wäldern gelebt hätten und wenn sie ihre gemalten Tafeln oder Bildwerke nicht auf dem Marktplatz ausgestellt hätten, damit sie jeder sehen und kritisieren konnte". ${ }^{65}$ Auch wenn es im Vorfeld und dann während der Querelle des Anciens et des Modernes zahlreiche, vehemente Gegenstimmen zu dieser Extremposition gab, so setzte sich diese letzten Endes bekanntlich doch durch.

Diese großen kulturhistorischen Einsichten werden bei Leonardo da Vinci wieder auf die Praxis, auf die einzelnen Werkstätten und kleinsten aemulativen Einheiten, die Meister-Schüler-Verhältnisse nämlich, heruntergebrochen: „Derjenige Schüler ist arm dran, der nicht seinen Lehrer übertrifft" ${ }^{66}$ Andererseits kann gescheiterte aemulatio das Ende eines Künstlers bedeuten - so wenn (zumindest in der Schilderung Paolo Giovios) Pietro Perugino, der in der Sixtinischen Kapelle den Wettstreit mit seinen Malerkollegen noch gewonnen hatte, am überragenden Vorbild eines Leonardo da Vinci, Raffael und Michelangelo verzweifelte und seine Fantasie „unfruchtbar “ wurde. ${ }^{67}$ Zum Glück scheinen in der Praxis aber die zwei Extremreaktionen auf solchen Wettstreit, wie sie die Künstleranekdoten von der Antike bis zur Frühen Neuzeit zahlreich kennen, die Ausnahme geblieben zu sein: Weder nahm sich jeder übertroffene Lehrer das Leben, noch brachte er seinen Schüler aus Neid um, wie es bereits Daidalos mit seinem talentierten Neffen Talos getan haben soll. ${ }^{68}$

Die aemulativen Ästhetiken können ab der Mitte des 16. Jahrhunderts sogar auch dazu führen, die Bildkünste und insbesondere die Malerei als einen 'Gewaltakt', die Maler als 'Soldaten' oder 'Degenhelden' und das Aufeinandertreffen unterschiedlicher künstlerischer Positionen als 'Kampf' zu beschreiben oder sogar als Kunsttheorie in Bildern zu visualisieren erinnert sei nur an den beliebten Mythos vom blutigen Wettstreit des Apoll und Marsyas. ${ }^{69}$ Der konkurrierende Vergleich entweder der verschiedenen

65 Enenkel 1995, $284 \mathrm{f}$. (II, 23).

66 Richter 1939, 308 (Nr. 498: „Tristo è quel discepolo che non ava[n]za il suo maestro").

67 Giovio 1999, 202-205: „[...] frustra Perusinus, meliora aemulando atque observando, partem dignitatem retinere conatus est, $[\ldots]$ sterilitate ingenii recidere cogeretur, $[\ldots]^{\prime \prime}$.

68 Kris/Kurz 1980 .

69 Weiterhin ließe sich auf eine umstrittene kunsttheoretische Kampf-Darstellung verweisen, ein Relief Hans Dauchers von 1522, das Dürer möglicherweise im Zweikampf mit Apelles (oder auch nur des Neides bzw. des Lasters) zeigt, Eser 1996; zu einem Gemälde Guido Renis siehe Pfisterer 2008. 
künstlerischen Medien Dichtung, Bildkünste, Musik usw., oder aber der Bildkünste untereinander (vor allem Malerei vs. Bildhauerkunst) wird mit Termini des Streits und der Auseinandersetzung bezeichnet - als disputà sulla maggioranza (bzw. nobiltà) delle arti, als combattimento, contesa usw. Höhepunkte dieser Entwicklung hin zu einer neu akzentuierten Ästhetik des aggressiv-gewaltsamen Wettstreits stellen dann im 17. Jahrhundert die Selbstbildnisse von Malern als bravi und 1665 Sebastiano Mazzonis Gedicht zur Pittura guerriera dar, ein parodiertes Epos, in dem die Rolle der gegeneinander kämpfenden Ritter von den verfeindeten Malerschulen von Florenz/Rom und der Lombardei/Venedigs eingenommen werden. ${ }^{70}$

(Wett-)Streit ist jedenfalls Motor historischen Wandels. Man kann wie Vasari - Geschichte als Folge von Überbietungen schreiben. Der Humanismus entwirft sich als Geschichte des Fortschritts auf Grund des Rückgriffs auf ein nachzuahmendes und zu überbietendes Modell. Die in der römischen Antike noch an der Peripherie gelegenen Länder des westlichen und nördlichen Europa treten mit ihren eigenen Kulturen in den Wettstreit ein. ${ }^{71}$ Im Bereich von Wissenschaft und Technik wird Fortschritt durch Überbietung auf Dauer gestellt (Bacon).

Die aemulatio der Renaissance mündet gegen Ende des 17. Jahrhunderts in die Querelle des Anciens et des Modernes, ${ }^{72}$ für die es seit dem 16. Jahrhundert viele Vorläufer gibt. Es wird häufig vergessen, dass Querelle Krieg bedeutet, Krieg der Kulturen, nicht nur der Literatur und der Schönen Künste, sondern auch der Wissenschaften, Techniken und zivilisatorischen Errungenschaften. ${ }^{73}$ Voltaires Siècle de Louis XIV ist der Versuch eines Kompromisses, der Entwurf von zweimal zwei Blütezeiten, zwei antiken, zwei modernen, deren Vorzüge auf unterschiedlichen Gebieten liegen. Der Krieg kann nur unentschieden ausgehen, denn man entdeckt, dass man Unvergleichbares vergleicht, dass Fortschritt und Vollkommenheit nicht deckungsgleich sind. So stößt man auf die Historizität der Kulturen. ${ }^{74}$ Historische Relativität hatte aber schon Erasmus in seinem Ciceronianus betont, als es ihm darum ging, den Wettstreit zwischen unterschiedlichen Fraktionen der Nachahmung der Antike zu scheiden. ${ }^{75}$

70 Vgl. Rossi, M. 1999, Mazzoni 2008 und Suthor 2010.

71 Robert 2002; ders. 2003; ders. 2007.

72 Jauß 1964; Kuhnle 2005.

73 Mazauric 2007.

74 Jauß 1964 u. 1967.

75 Pigmann 1980; Müller, J.-D. 1999. 
Der Band enthält die ausgearbeiteten Vorträge des Kolloquiums. Hinzu kamen die Aufsätze von Barbara Mahlmann-Bauer, Regina Toepfer und Maurice Saß sowie ein zweiter Aufsatz von Fabian Jonietz. In zwei Fällen wurde der Beitrag auf Grund der Diskussionen während der Tagung neu konzipiert. Jedes Mal ging der Publikation ein intensiver Gedankenaustausch über einen erweiterten aemulatio-Begriff voraus.

Es gilt vielfältigen Dank abzustatten: dem Vorstand des SFB - besonders seinem Sprecher Andreas Höfele -, der die Finanzierung des Kolloquiums ermöglichte und den Tagungsband in sein Publikationsprogramm aufnahm; dem Münchner Center for Advanced Studies, das seine Räume zur Verfügung stellte; den Mitherausgebern Anna Kathrin Bleuler und Fabian Jonietz, die zusammen mit uns, mit Jan Hon, Sylvia Brockstieger und Semjon A. Dreiling, sich um die Abstimmung der Beiträge und ihre Ausrichtung auf die zentrale Fragestellung bemühten, wobei die oft schwierige Koordination der Arbeiten in den Händen von Jan Hon lag; den Hilfskräften Simone Ketterl, Ulrike Keuper, Kathrin Lukaschek, Fabian Prechtl, Johanna Pulz und Anna Steger, die über die redaktionelle Einrichtung wachten; dem Publikationsbüro, das die Druckvorlage erstellte; den Helfern während der Durchführung des Kolloquiums.

Jan-Dirk Müller und Ulrich Pfisterer

\section{Bibliographie}

\section{Quellen}

[Bartolini, Ricardo (1515)]: Odeporicon id est Itinerarium Reuerendissimi in Christo patris et Domini D. Mathei Sancti Angeli Cardinalis Gurcensis coadiutoris Saltzburgensis Generalisque Imperii locumtenentis, Quaeque in conuentu Maximiliani Caesaris Augusti Serenissimorumque regum Vladislai Sigismundi ac Ludouici, memoratu digna gesta sunt per Riccardum Bartholinum perusinum redita. Cum Gratia et priuilegio. Wien: [Hieronymus Vietor].

[Cortese, Paolo/Poliziano, Angelo (1952)]: „Briefwechsel“, in: Garin, Eugenio (Hrsg.): Prosatori latini del Quattrocento. Mailand: Riccardi, 902-911.

Decembrio, Angelo C. (2002): De politia litteraria. Hrsg. von Norbert Witten. München/Leizpig: Saur.

Dęmontiosius, Ludovicus (1585): „Commentarii de sculptura, et pictura antiquorum", in: ders.: Gallus Romae Hospes. Rom: Giovanni Gigliotti.

Dufresnoy, Charles-Alphonse (2005): De arte graphica. Hrsg. von Christopher Allen, Yasmin Haskell und Frances Muecke. Genf: Droz. 
Erasmus von Rotterdam, Desiderius (1972): Dialogus cui titulus Ciceronianus sive de optimo dicendi genere. Übersetzt, eingeleitet und mit Anmerkungen versehen von Theresia Payr. Darmstadt: Wissenschaftliche Buchgesellschaft (= Ausgewählte Schriften in acht Bänden, 7).

Fischart, Johann (1588): Ernewerte Beschreibung/der Wolgedenckwuirdigen Alten vnd warhafften verwunderlichen Geschicht. Vom Herren Petern von Stauffenberg genant Diemringer [...]. Darzu ein außfülicher Bericht vnd Vorred gethan worden [...]. Magdeburg.

Fischart, Johann (1895): „Das Philosophisch Ehzuchtbüchlein“, in: Hauffen, Adolf (Hrsg.): Johann Fischarts Werke. Eine Auswahl. Bd. 3. Stuttgart: Union Dt. Verl.-Ges. (= Deutsche National-Litteratur, 18, 3), 115-332.

Giovio, Paolo (1999): Scritti d'Arte. Lessico ed ecfrasi. Hrsg. von Sonia Maffei. Pisa: Scuola Normale Superiore.

Holtzwart, Mathias (2006): Emblematum Tyrocinia. Mit einem Vorwort über Ursprung, Gebrauch und Nutz der Emblematen von Johann Fischart und 72 Holzschnitten von Tobias Stimmer. Hrsg. von Peter von Düffel und Klaus Schmidt. Stuttgart: Reclam.

Leonardo da Vinci ( $\left.{ }^{2} 1939\right)$ : The Literary Works of Leonardo da Vinci. Compiled and edited from the Original Manuscripts. Hrsg. Von Jean Paul Richter. London / New York/Toronto: Oxford University Press.

Macioce, Stefania (Hrsg.) (2003): Michelangelo Merisi da Caravaggio. Fonti e documenti 1532-1724. Rom: Bozzi.

Marsuppini, Carlo (1940): „Consolatio de obitu matris“. Hrsg. von Pier G. Ricci, in: La Rinascita 3, 362-433.

Mazzoni, Sebastiano (2008): 'La pittura guerriera' e altri versi sull'arte. Hrsg. von Massimiliano Rossi. Caselle di Sommacampagna: Cierre Edizioni.

Opitz, Martin (2002): Buch von der Deutschen Poeterey (1624). Studienausgabe. Mit dem Aristarch (1617) und den Opitzischen Vorreden zu seinen Teutschen Poemata (1624 und 1625) sowie der Vorrede zu seiner Übersetzung der Trojanerinnen (1625). Hrsg. von Herbert Jaumann. Stuttgart: Reclam.

Pico della Mirandola, Gian Francesco/Bembo, Pietro (1954): Le Epistole 'de imitatione. Hrsg. von Giorgio Santangelo. Florenz: Leo S. Olschki.

Valla, Lorenzo (1962): „Oratio habita in principio sui studii die XVIII. Octobris MCCCCLV", in: ders.: Opera Omnia. Hrsg. von Eugenio Garin. Turin: Bottega d'Erasmo, Bd. 2, 282. [Nachdruck der Ausgabe Basel 1540].

Vergerio, Pietro Paulo (1917-1918): „De ingenuis moribus et liberalibus studiis adulescentiae libellus in partes duas", in: Attilio Gnesotto (Hrsg.): Atti e Memorie della Reale Accademia di Scienze Lettere ed Arti in Padova. N.S. 34, $75-157$.

Villani, Filippo (1997): De origine civitatis Florentie et de eiusdem famosis civibus. Hrsg. von Giuliano Tanturli. Padua: Antenore.

Zincgref, Julius Wilhelm (1879): Auserlesene Gedichte Deutscher Poeten (1624). Gesammelt von Julius Wilhelm Zinkgref. Hrsg. von Wilhelm Braune. Halle a.S.: Niemeyer (= Neudrucke deutscher Litteraturwerke des XVI. und XVII. Jahrhunderts, 15).

Zuccari, Federico (1961): Scritti d'arte. Hrsg. von Detlef Heikamp. Florenz: Il Cenacolo (= Fonti per lo studio della storia dell'arte inedite o rare, 1). 


\section{Forschungsliteratur}

Acidini, Cristina (Hrsg.) (2009): Innocente e calunniato. Federico Zuccari (1539/ 40-1609) e le vendette d'artista. Florenz/Mailand: Giunti.

Ames-Lewis, Francis/Joannides, Paul (Hrsg.) (2003): Reactions to the Master. Michelangelo's effect on art and artists in the sixteenth century. Aldershot u. a.: Ashgate Publishing.

Antragsbuch (2007): Ludwig-Maximilians-Universität München. Sonderforschungsbereich 573. Pluralisierung und Autorität in der Frühen Neuzeit [15.17. Jahrhundert]. Finanzierungsantrag für den dritten Förderzeitraum [1. Januar 2008-31. Dezember 2011].

Baader, Hannah (2007): „Ernste Spiele, 1441: Niccolò della Luna und Leon Battista Alberti über Wettstreit, Freundschaft, Neid und Kunst", in: dies. / Müller Hofstede, Ulrike u. a. (Hrsg.): Im Agon der Künste. Paragonales Denken, ästhetische Praxis und die Diversität der Sinne. München: Fink, $32-$ 49.

Baader, Hannah / Müller Hofstede, Ulrike u. a. (2007) (Hrsg.): Im Agon der Künste. Paragonales Denken, ästhetische Praxis und die Diversität der Sinne. München: Fink.

Barbieri, Costanza (2005): „The Competition between Raphael and Michelangelo and Sebastiano's Role in It", in: Hall, Marcia B. (Hrsg.): The Cambridge Companion to Raphael. Cambridge: Cambridge University Press, 141-164.

Bauer, Bảbara (1992): Art. „Aemulatio“, in: Ueding, Gert (Hrsg.): Historisches Wörterbuch der Rhetorik. Bd. 1. Tübingen: Niemeyer, 141-187.

Baxandall, Michael (1963): „A dialogue on art from the court of Leonello d'Este: Angelo Decembrio's 'De Politica Letteraria, Pars LXVIII'", in: Journal of the Warburg and Courtauld Institutes 26, 304-326.

Baxandall, Michael (1971): Giotto and the Orators. Humanist observers of painting in Italy and the discovery of pictorial composition, 1350-1450. Oxford: Oxford University Press.

Bjaï, Denis (1997): „De l'épopée nationale à l'Histoire auvergnate: Ronsard et Jean de Boyssières", in: Nouvelle Revue du XVIe siècle 15, 54-71.

Bleuler, Anna Kathrin (2009): „Imitatio veterum - imitatio modernorum“, in: Daphnis 38, 527-554.

Bolland, Andrea L. (1996): „Art and humanism in early Renaissance Padua: Cennini, Vergerio and Petrarch on imitation“, in: Renaissance Quarterly 49, 469-487.

Bornscheuer, Marion (2008): „Geglückte 'aemulatio'. Dürers Mantegna-Rezeption und seine Rückbeeinflussung der italienischen Graphik", in: Jahrbuch der Staatlichen Kunstsammlungen in Baden-Württemberg 45, 31-48.

Brown, Louise B. (2001): „Die schwarzen Schwingen des Neides. Konkurrenz, Rivalität und Vorbild", in: dies. (Hrsg.): Die Geburt des Barock. Stuttgart: Belser, 248-273.

Burckhardt, Jacob (2006): Die Kunst der Renaissance. Band 1: Geschichte der Renaissance in Italien. Die Malerei nach Inhalt und Aufgabe. Randglossen zur Sculptur der Renaissance. Hrsg. von Maurizio Ghelardi u. a. München u. a.: C.H. Beck (= Kritische Gesamtausgabe, 16). 
Burioni, Matteo (2011): „Der Künstler als Champion. Kunsttheoretische Figuren des Grundes in der frühen Neuzeit", in: Busche, Hubertus (Hrsg.): Departure for Modern Europe. A Handbook of Early Modern Philosophy (1400-1700). Hamburg: Meiner, 954-968.

Bußmann, Britta/Hausmann, Albrecht u.a. (Hrsg.) (2005): Übertragungen. Formen und Konzepte von Reproduktion in Mittelalter und Früher Neuzeit. Berlin u.a.: De Gruyter (= Trends in medieval philology, 5).

Campanelli, Maurizio (2001): Polemiche e filologia ai primordi della stampa. Le Observationes di Domizio Calderini. Roma: Edizioni di Storia e Letteratura (= Sussidi Euditi, 54).

Campbell, Stephen J. u.a. (Hrsg.) (2004): Artists at Court. Image-making and identity 1300-1550. Boston/Chicago: University of Chicago Press.

Cave, Terence (1979): The Cornucopian Text. Problems of Writing in the French Renaissance. Oxford: Clarendon Press.

Clifton, James (1996): „Vasari on Competition“, in: Sixteenth-Century Journal 27, $23-41$.

Cole, Michael W. (2011): Ambitious Form. Giambologna, Ammanati, and Danti in Florence. Princeton/Oxford: Princeton University Press.

Cropper, Elizabeth (2005): The Domenichino Affair. Novelty, Imitation, and Theft in Seventeenth-Century Rome. New Haven/London: Yale University Press.

Csürös, Klára (1999): Variétés et vicissitudes du genre épique de Ronsard à Voltaire. Paris: Champion.

Delaney, Susan (1993-1994): „L'Olive and the Poetics of Rivalry“, in: Classical and Modern Literature 14, 183-196.

Dempsey, Charles (1993): „Idealism and Naturalism in Rome around 1600“, in: De Luca, Elena (Hrsg.): Il classicismo: medioevo, rinascimento, barocco. Bologna: Nuova Alfa editoriale, 233-243.

Donato, Maria M. (2003): „Veteres et novi, externi et nostri'. Gli artisti di Petrarca: per una rilettura", in: Quintavalle, Arturo C. (Hrsg.): Medioevo: immagine e racconto. Mailand: Mondadori Electa, 433-455.

Drücke, Simone (2001): Humanistische Laienbildung um 1500. Das Übersetzungswerk des rheinischen Humanisten Johann Gottfried. Göttingen: Vandenhoeck \& Ruprecht (= Palaestra, 312). (DVR)

Dundas, Judith (1998): „A mutual emulation'. Sidney and the painting of the ancients", in: Bremmer, Rolf H. Jr. (Hrsg.): Franciscus Junius F.F and His Circle. Amsterdam u. a.: Rodopi, $71-92$.

Enenkel, Karl A. E. (1995): Kulturoptimismus und Kulturpessimismus in der Renaissance. Studie zu Jacob Canters 'Dyalogus de Solitudine'. Mit kritischer Textausgabe und deutscher Übersetzung. Frankfurt a.M. u. a.: Peter Lang.

Erben, Dietrich (2002): „Das Bild des 'Condottiere' bei Jacob Burckhardt: Militarisierung und Renaissancerezeption um 1900“, in: Ghelardi, Maurizio/Seidel, Max (Hrsg.): Jacob Burckhardt. Storia della cultura, storia dell'arte. Venedig: Marsilio, $147-158$.

Eser, Thomas (1996): Hans Daucher. Augsburger Kleinplastik der Renaissance. München/Berlin: Deutscher Kunstverlag.

Fehrenbach, Frank (2011): Rezension über Nagel, Alexander/ Wood, Christopher S. (Hrsg.) (2010): Anachronic Renaissance. New York: Zone Books, in: Kunstform 
12/3. URL: <http://www.arthistoricum.net/index.php?id=2768xausgabe $=2011 \_038$ review_id=18816> [letzter Zugriff: 8. 8.2011)].

Fera, Vincenzo (1999): „Il problema dell' imitatio tra Poliziano e Cortesi“, in: Fera, Vincenzo / Guida, Augusto (Hrsg.): Vetustatis indagator. Scritti offerti a Filippo Di Benedetto. Messina: Universita degli Studi di Messina, 155-181.

Fera, Vincenzo (2004): "L'imitatio umanistica“, in: Perini Bernardi, Giorgio (Hrsg.): Il latino nell'età dell'Umanesimo. Firenze: Olschki, 17-33.

Ferguson, Gary (2003): „Reviving Epic in Renaissance France: Ronsard, Jamyn, and other Homers", in: Ferguson, Gary / Hampton, Catherine (Hrsg.): (Re)Inventing the Past: Essays on French Early Modern Culture, Literature and Thought in Honour of Ann Moss. Durham: University of Durham, 125-152. Flood, John Lewis (2006): Poets laureate in the Holy Roman Empire. A bio-bibliographic handbook. Berlin: De Gruyter.

Flood, John Lewis (2008): „Das Bild des Poeta laureatus in Deutschland und England um 1500", in: McLelland, Nicola / Schiewer, Hans-Jochen / Schmitt, Stefanie (Hrsg.): Humanismus in der deutschen Literatur des Mittelalters und der Frühen Neuzeit. XVIII. Anglo-German Colloquium Hofgeismar 2003. Tübingen: Niemeyer, 399-428.

Föcking, Marc/Müller, Gernot M. (Hrsg.) (2007): Abgrenzung und Synthese. Lateinische Dichtung und volkssprachliche Traditionen in Renaissance und Barock. Lateinische Dichtung und volkssprachliche Tradition von der Renaissance bis zum Neoklassizismus. Heidelberg: Winter (= Germanisch-Romanische Monatsschrift, Beiheft 31).

Foucault, Michel (1973): Archäologie des Wissens. Frankfurt a.M.: Suhrkamp.

Gilbert, Neal W. (1977): „A letter of Giovanni Dondi dall'Orologio to Fra' Guglielmo Centueri. A fourteenth-century episode in the quarrel of the ancients and the moderns", in: Viator 8, 299-346.

Gmelin, Hermann (1932): „Das Prinzip der Imitatio in den romanischen Literaturen der Renaissance", in: Romanische Forschungen 46, 83-360.

Goffen, Rona (2002): Renaissance Rivals. Michelangelo, Leonardo, Raphael, Titian. New Haven/London: Yale University Press.

Goldthwaite, Richard A. (1993): Wealth and the Demand for Art in Italy 1300-1600. Baltimore u. a.: Johns Hopkins University Press.

Gorni, Guglielmo (1972): „Storia del Certame Coronario“, in: Rinascimento ser. 2, $12,135-181$.

Green, Louis (1990): „Galvano Fiamma, Azzone Visconti and the revival of the classical theory of magnificence", in: Journal of the Warburg and Courtauld Institutes 53, 98-113.

Greene, Thomas M. (1982): The Light in Troy. Imitation and Discovery in Renaissance Poetry. New Haven/London: Yale University Press.

Grenzmann, Ludger/Grubmüller, Klaus u.a. (Hrsg.) (2004): Die Präsenz der Antike im Übergang vom Mittelalter zur Frühen Neuzeit. Bericht über Kolloquien der Kommission zur Erforschung der Kultur des Spätmittelalters 1999 bis 2002. Göttingen: Vandenhoeck \& Ruprecht (= Philologisch-Historische Klasse: Dritte Folge, 263).

Herrmann-Fiore, Kristina (1996): „Die Freien Künste im Altertum und in der Neuzeit. Ein Wettstreit, dargestellt von Alessandro Tassoni im Jahr 1620“, in: 
von Flemming, Victoria/ Schütze, Sebastian (Hrsg.): Ars Naturam Adiuvans. Festschrift für Matthias Winner. Mainz: Zabern, 381-400.

Hoppe, Stephan/Müller, Matthias / Nußbaum, Norbert (Hrsg.) (2008): Stil als Bedeutung. Stil als Bedeutung in der nordalpinen Renaissance - Wiederentdeckung einer methodischen Nachbarschaft. 2. Sigurd Greven-Kolloquium zur Renaissanceforschung. Regensburg: Schnell \& Steiner.

Ilchman, Frederick (Hrsg.) (2009): Titian, Tintoretto, Veronese. Rivals in Renaissance Venice. Franham Surrey: Lund Humphries Publishers.

Irle, Klaus (1997): Der Ruhm der Bienen. Das Nachahmungsprinzip der italienischen Malerei von Raffael bis Rubens. Münster u. a.: Waxmann (= Internationale Hochschulschriften, 230).

Jardine, Lisa (1996): Wordly Goods. A New History of the Renaissance. London: W. W. Norton \& Company.

Jauß, Hans Robert (1964): Asthetische Normen und geschichtliche Reflexion in der 'Querelle des anciens et des modernes'. München: Eidos Verlag.

Jauß, Hans Robert (1967): „Fr. Schlegels und Fr. Schillers Replik auf die ‘Querelle des Anciens et des Modernes' ", in: Friedrich, Hugo/Schalk, Fritz (Hrsg.): Europäische Aufklärung. Herbert Dieckmann zum 60. Geburtstag. München: Fink, $117-140$.

Joyce, Hetty (1992): „Grasping at shadows. Ancient paintings in Renaissance and Baroque Rome", in: The Art Bulletin 74, 219-246.

Kaminski, Nicola (1998): Art. „Imitatio“, in: Ueding, Gert (Hrsg.): Historisches Wörterbuch der Rhetorik. Bd. 4. Tübingen: Niemeyer, 235-285.

Kanz, Roland (2011): „Künstlerstreit in der frühen Neuzeit als Movens für Kreativität", in: Busche, Hubertus/Hessbrueggen-Walter, Stefan (Hrsg.): Departure for Modern Europe. A handbook of early modern philosophy (1400-1700). Hamburg: Felix Meiner, 927-938.

Kaschek, Bertram (2009): „Weder römisch, noch antik?' Pieter Bruegels Verleumdung des Apelles in neuer Deutung", in: Kamecke, Gernot/Klein, Bruno u. a. (Hrsg.): Antike als Konzept. Lesarten in Kunst, Literatur und Politik. Berlin: Lukas Verlag, 167-179.

Koch, Nadja J. (2004): „Phidias und Polyklet im Agon. Die neueren archäologischen und kunsthistorischen Forschungen zur Rezeption griechischer Bildhauer in der Renaissance", in: International Journal of the Classical Tradition $11,244-265$.

Koerner, Joseph L. (1993): The Moment of Self-Portraiture in German Renaissance Art. Chicago: University of Chicago Press.

Kris, Ernst/Kurz, Otto ([1934] 1980): Die Legende vom Künstler. Ein geschichtlicher Versuch. Frankfurt a.M.: Suhrkamp (= Edition Suhrkamp N.F. 1034, 34).

Küenzlen, Franziska (2005): Verwandlungen eines Esels. Apuleius' 'Metamorphoses' im frühen 16. Jahrhundert. Der Kommentar Filippo Beroaldos d.Ä - Die Übersetzungen von Johann Sieder, Guillaume Michel, Diego López de Cortegana und Agnolo Firenzuola - Der Schelmenroman Lazarillo de Tormes. Heidelberg: Winter (= Germanisch-romanische Monatsschrift, Beiheft, 25). 
Kühłmann, Wilhelm/Neuber, Wolfgang (Hrsg.) (1994): Intertextualität in der frühen Neuzeit. Studien zu ihren theoretischen und praktischen Perspektiven. Frankfurt a.M. u. a.: Lang (= Frühneuzeitstudien, 2).

Kühlmann, Wilhelm (Hrsg.) (2011): Julius Wilhelm Zincgref und der Heidelberger Späthumanismus. Zur Blüte- und Kampfzeit der calvinistischen Kurpfalz. Ubstadt-Weiher u. a.: Regionalkultur (= Mannheimer historische Schriften, 5).

Kuhnle, Till R. (2005): Art. „Querelle“, in: Ueding, Gert (Hrsg.): Handwörterbuch der Rhetorik. Bd. 7. Tübingen: Niemeyer, 503-532.

Mai, Ekkehard/Wettengl, Kurt (Hrsg.) (2002): Wettstreit der Künste. Malerei und Skulptur von Dürer bis Daumier. Wolfratshausen: Edition Minerva.

Mazauric, Simone (2007): Fontenelle et l'invention de l'histoire des sciences à l'aube des Lumières. Paris: Fayard (= Histoire de la pensée).

Metzger, Christoph (2010): „'es muoss ein zeserlin haben': Überlegungen zur Funktion des Naturalismus im späten Mittelalter und ein Jesuskind von Nikolaus Gerhaert von Leyden“, in: Tacke, Andreas/Heinz, Stefan (Hrsg.): Menschenbilder. Beiträge zur Altdeutschen Kunst. Petersberg: Michael Imhof, 57-80.

Michalsky, Tanja (2005): „Schichten der Erinnerung. Tradition, Innovation und 'Aemulatio' in der neapolitanischen Sepulkralplastik“, in: Borgolte, Michael u. a. (Hrsg.): Memoria. Ricordare e dimenticare nella cultura del medioevo. Bologna: Il Mulino (= Annali dell' Istituto Storico Italo-Germanico in Trento, Contributi, 15), 99-131.

Middeldorf Kosegarten, Antje (1980): „The origins of artistic competitions in Italy (forms of competition between artists before the contest for the Florentine Baptistery doors won by Ghiberti in 1401)", in: Krautheimer, Richard (Hrsg.): Lorenzo Ghiberti nel suo tempo. Atti del convegno internazionale di studi. Bd. 1. Florenz: Leo S. Olschki, 167-186.

Moffitt, John F. (2004): Caravaggio in Context. Learned naturalism and Renaissance humanism. Jefferson, $\mathrm{NC}$ u. a.: McFarland.

Müller, Jan-Dirk (1982): Gedechtnus. Literatur und Hofgesellschaft um Maximilian I. München: Fink (= Forschungen zur Geschichte der älteren deutschen Literatur, 2).

Müller, Jan-Dirk (1999): „Warum Cicero? Erasmus' 'Ciceronianus' und das Problem der Autorität", in: Scientia Poetica 3, 20-46.

Müller, Jan-Dirk (2007a): „Fischarts Gegenkanon. Komische Literatur im Zeichen der imitatio", in: ders./Robert, Jörg (Hrsg.): Maske und Mosaik. Poetik, Sprache, Wissen im 16. Jahrhundert. Berlin: LIT (= P \& A, 11), 281-321.

Müller, Jan-Dirk (2007b): „Formung der Sprache und Formung durch Sprache. Zur anthropologischen Interpretation des imitatio-Konzepts", in: ders./ Robert, Jörg(Hrsg.): Maske und Mosaik. Poetik, Sprache, Wissen im 16. Jahrhundert. Berlin: LIT (= P \& A, 11), 159-199.

Müller, Jan-Dirk (2009): „Maximilian und die Hybridisierung frühneuzeitlicher Hofkultur. Zum Ludus Dianae und der Rhapsodia des Konrad Celtis", in: Jahrbuch der Oswald von Wolkenstein-Gesellschaft 17 (2008/2009), 3-21.

Müller, Jan-Dirk (2011): „Die Frühe Neuzeit in der Literaturgeschichtsschreibung", in: Lepper, Marcel/Werle, Dirk (Hrsg.): Entdeckung der frühen Neuzeit. Konstruktionen einer Epoche der Literatur- und Sprachgeschichte seit 
1750. Stuttgart: Hirzel (= Beiträge zur Geschichte der Germanistik, 1), 1632.

Müller, Jan-Dirk [im Druck]: „Präsenz des Heils und Repräsentation. Zur Alterität des Geistlichen Spiels (mit einem Nachwort zu anderen Formen des mittelalterlichen 'Dramas')", in: Becker, Anja/Mohr, Jan (Hrsg.): Alterität als Leitkonzept für historisches Interpretieren. Berlin: Akademie Verlag (= Deutsche Literatur. Studien und Quellen).

Müller, Jürgen (1993): Concordia Pragensis. Karel van Manders Kunsttheorie im Schilder-Boeck. München: Oldenbourg.

Müller, Jürgen (2007): „Italienverehrung als Italienverachtung. Hans Sebald Behams Jungbrunnen von 1536 und die italienische Kunst der Renaissance", in: Helas, Philine/Polte, Maren u.a. (Hrsg.): Bild-Geschichte. Festschrift für Horst Bredekamp. Berlin: Akademie-Verlag, 309-318.

Nagel, Alexander/Wood, Christopher S. (Hrsg.) (2010): Anachronic Renaissance. New York: Zone Books.

Pfisterer, Ulrich (1999): „Phidias und Polyklet von Dante bis Vasari. Zu Nachruhm und künstlerischer Rezeption antiker Bildhauer in der Renaissance", in: Marburger Jahrbuch für Kunstwissenschaft 26, 61-97.

Pfisterer, Ulrich (2002): Donatello und die Entdeckung der Stile 1430-1445. München: Hirmer (= Römische Studien der Bibliotheca Hertziana, 17).

Pfisterer, Ulrich (2003): Art. „Paragone“, in: Ueding, Gert (Hrsg.): Historisches Wörterbuch der Rhetorik. Bd. 6. Tübingen: Niemeyer, 528-546.

Pfisterer, Ulrich (2008): „Kampf der Malerschulen - Guido Renis Lotta dei Putti und die 'Caravaggisten'", in: Mitteilungen des Sonderforschungsbereich 573 'Pluralisierung und Autorität in der Frühen Neuzeit' 4, 16-26.

Pfisterer, Ulrich (2011): Art. „Schule“, in: ders. (Hrsg.): Metzler Lexikon Kunstwissenschaft. Ideen, Methoden, Begriffe. 2. erweiterte Aufl. Stuttgart/Weimar: Metzler, 402-406.

Pfisterer, Ulrich/Wimböck, Gabriele (Hrsg.) (2011): 'Novità' - Neuheitskonzepte in den Bildkünsten um 1600. Zürich/Berlin: Diaphanes.

Pigman, George W. (1979): „Imitation and the Renaissance sense of past. The reception of Erasmus' 'Ciceronianus'", in: Journal of Medieval and Renaissance Studies 9, 155-177.

Pigman, George W. (1980): „Versions of Imitation in the Renaissance“, in: Renaissance Quarterly 33, 1-32.

Plank, Birgit (2004): Johann Sieders Übersetzung des 'Goldenen Esels' und die frühe deutschsprachige 'Metamorphosen'-Rezeption. Ein Beitrag zur Wirkungsgeschichte von Apuleius' Roman. Tübingen: Niemeyer (= Frühe Neuzeit, 92).

Pochat, Götz (1987): „Imitatio und Superatio - das Problem der Nachahmung aus humanistischer und kunsttheoretischer Sicht", in: Meyer zur Capellen, Jürg/Oberreuter-Kronabel, Gabriele (Hrsg.): Klassizismus. Epoche und Probleme. Festschrift für Erik Forssman zum 70. Geburtstag. Hildesheim/Zürich/New York: Olms, 317-335.

Prochno, Renate (2006): Konkurrenz und ihre Gesichter in der Kunst. Wettbewerb, Kreativität und ihre Wirkungen. Berlin: Akademie-Verlag.

Quint, David (1993): Epic and Empire: Politics and Generic Form from Virgil to Milton. Princeton: Princeton University Press. 
Reilly, Patricia L. (2010): „Raphael's 'Fire in the Borgo' and the Italian Pictorial Vernacular", in: The Art Bulletin 92, 308-325.

Reinhardt, Volker (2004): „Die Renaissance-Tyrannis als Laboratorium der Moderne: Epochenwandel bei Jacob Burckhardt", in: Cesana, Andreas/Gossman, Lionel (Hrsg.): Begegnungen mit Jacob Burckhardt. Vorträge in Basel und Princeton zum hundertsten Todestag. Basel/München: Schwabe (= Beiträge zu Jacob Burckhardt, 4), 449-466.

Ricci, Pier Girogio (1974), "La tradizione dell'invettiva tra il Medioevo e l'Umanenismo", in: Lettere Italiane 26, 405-414.

Rigolot, François (1988): „Between Homer and Virgil: Mimesis and Imitatio in Ronsard's Epic Theory", in: Horowitz, Maryanne Cline u.a. (Hrsg.): Renaissance Rereadungs. Intertext and Context. Urbala/Chicago: University of Illinois Press, 67-79.

Robert, Jörg (2002): „'Carmina Pieridum nulli celebrata priorum’. Zur Inszenierung von Epochenwende im Werk des Conrad Celtis", in: Beiträge zur Geschichte der deutschen Sprache und Literatur 124, 92-121.

Robert, Jörg (2003): Konrad Celtis und das Projekt der deutschen Dichtung. Studien zur humanistischen Konstitution von Poetik, Philosophie, Nation und Ich. Tübingen: Niemeyer (= Frühe Neuzeit, 76).

Robert, Jörg (2007): „Deutsch-lateinische Dornen: Paulus Melissus Schede und die Pluralisierung der späthumanistischen Poetik zwischen Latinität und Volkssprache[n]", in: Föcking, Marc/Müller, Gernot M. (Hrsg.): Abgrenzung und Synthese. Lateinische Dichtung und volkssprachliche Traditionen in Renaissance und Barock. Heidelberg: Winter (= Germanisch-Romanische Monatsschrift, Beiheft 31), 207-229.

Rossi, Massimiliano (1999): „La peinture guerrière. Artists et paladins à Venise au XVIIIe siècle", in: Careri, Giovanni (Hrsg.): La Jérusalem délivrée du Tasse: poésie, peintrue, musique, ballet. Paris: Klincksieck (= Conférences et colloques, Louvre), 67-108.

Rossi, Vittorio (1992): Il Quattrocento. Padova: Piccin Nuova Libraria (= Storia letteraria d'Italia Nuova edizione, 6).

Schirrmeister, Albert (2003): Triumph des Dichters. Gekrönte Intellektuelle im 16. Jahrhundert. Köln: Böhlau (= Frühneuzeit-Studien N.F., 4).

Shearman, John K.G. ([1967] 1988): Manierismus. Das Künstliche in der Kunst. Frankfurt a.M.: Athenäum.

Silver, Isidore (1961): Ronsard and the Hellenic Renaissance in France. Bd. 1. St. Louis: Washington University.

Smyth, Craig H. ([1963] 1992): Mannerism and maniera. Eingeleitet von Elizabeth Cropper. Wien: IRSA (= Bibliotheca artibus et historiae).

Sohm, Philip (2001): Style in the Art Theory of Early Modern Italy. Cambridge u. a.: Cambridge University Press.

Stoichita, Victor I. ([1993] 1995): „Ars ultima: Bemerkungen zur Kunsttheorie des Manierismus", in: Bonnet, Anne-Marie/Kopp-Schmidt, Gabriele (Hrsg.): Kunst ohne Geschichte? Ansichten zu Kunst und Kunstgeschichte heute. München: C.H. Beck, 154-162.

Stoichita, Victor I. (1998): Das selbstbewußte Bild. Vom Ursprung der Metamalerei. München: Fink. 
Suthor, Nicola (2010): Bravura. Virtuosität und Mutwilligkeit in der Malerei der Frühen Neuzeit. München: Fink.

Tauber, Christine (2009): Manierismus und Herrschaftspraxis. Die Kunst der Politik und die Kunstpolitik am Hof von François $I^{r}$. Berlin: Akademie Verlag (= Studien aus dem Warburg-Haus, 10).

Thielemann, Andreas (1996): Phidias im Quattrocento. [Photomechanischer Abdruck der Diss. phil. Univ. Köln 1992].

Trinkaus, Charles (1987): „Antiquitas versus Modernitas': An humanist Polemic and its Resonance", in: Journal of the History of Ideas 48, 11-21.

Vermeylen, Filip (2003): Painting for the Market. Commercialization of art in Antwerp's Golden Age. Turnhout: Brepols (= Studies in European urban history, 2).

Vismara, Felice (1990): L'Invettiva, arma preferita dagli Umanisti nelle lotte private, nelle polemiche letterarie, politiche e religiose. Milano: Allegretti.

Warnke, Martin (1985): Hofkünstler. Zur Vorgeschichte des modernen Künstlers. Köln: Dumont (= DuMont's Bibliothek der Kunstwissenschaft).

Weissert, Caecilie (2008): „Nova Roma'. Aspekte der Antikenrezeption in den Niederlanden im 16. Jahrhundert", in: Artibus \& Historiae 29/58, 173-200.

Welzel, Barbara (2006): „Verhüllen und Inszenieren: zur performativen Praxis in frühneuzeitlichen Sammlungen“, in: Felfe, Robert/Lozar, Angelika (Hrsg.): Frühneuzeitliche Sammlungspraxis und Literatur. Berlin: Lukas Verlag, 109129.

Williams, Robert (1997): „Art, Theory and Culture in Sixteenth-Century Italy. From techne to metatechne". Cambridge: Cambridge University Press.

Wimböck, Gabriele (2008): „Unschuldige Blicke. Skizzen zur Kunstbetrachtung des 'Laien' in der Frühen Neuzeit", in: Albrecht, Stephan/Braesel, Michaela u. a. (Hrsg.): Kunst, Geschichte, Wahrnehmung. Strukturen und Mechanismen von Wahrnehmungsstrategien. München/Berlin: Deutscher Kunstverlag (= Münchener Universitätsschriften des Instituts für Kunstgeschichte, 7), 281 293.

Wittkower, Margot und Rudolf ([1963] 1989): Künstler - Außenseiter der Gesellschaft. Stuttgart: Klett-Cotta.

Zymner, Rüdiger (1995): Manierismus. Zur poetischen Artistik bei Johann Fischart, Jean Paul und Arno Schmidt. Paderborn/München: Schöningh.

Zymner, Rüdiger (1997): Art. „Argutia“, in: Weimar, Klaus (Hrsg.): Reallexikon der deutschen Literaturwissenschaft. Neubearbeitung des Reallexikons der deutschen Literaturgeschichte. Bd. 1. Berlin/New York: De Gruyter, 133-135. 